\title{
Phytochemical and Pharmacological Properties of the Genus Melodinus - A Review
}

\author{
Jin-He Jiang ${ }^{1-3}$, Wu-Di Zhang ${ }^{1}$ and Ye-Gao Chen $^{2}$ \\ ${ }^{1}$ Faculty of Energy and Environmental Science, ${ }^{2}$ Department of Chemistry, ${ }^{3}$ Department of Library, Yunnan Normal University, \\ Kunming 650500, PR China
}

*For correspondence: Email: jinhejiang@126.com, ygchen48@gmail.com; Tel: +86-871-5941089

\begin{abstract}
Melodinus is an important genus comprising of approximately 53 species of medicinal plants (Apocynaceae). Some species have been used in Chinese folk medicine for the treatment of meningitis in children, rheumatic heart diseases, and diuresis, as well as a decongestive against migraine and sinusitis. This paper is a review of the literature up to May 2015 and describes 263 compounds from 69 articles, and includes chemical constituents isolated from Melodinus, mainly indole alkaloids, quinoline alkaloids, dimeric alkaloids, terpenoids and other compounds. It is also hoped that an overview of their cytotoxic characteristics will further the development of new anti-cancer agents.
\end{abstract}

Keywords: Melodinus, Indole alkaloids, Dimeric alkaloids, Cytotoxicity, Medicinal plants

\begin{abstract}
Tropical Journal of Pharmaceutical Research is indexed by Science Citation Index (SciSearch), Scopus, International Pharmaceutical Abstract, Chemical Abstracts, Embase, Index Copernicus, EBSCO, African Index Medicus, JournalSeek, Journal Citation Reports/Science Edition, Directory of Open Access Journals (DOAJ), African Journal Online, Bioline International, Open-J-Gate and Pharmacy Abstracts
\end{abstract}

\section{INTRODUCTION}

Melodinus is a genus of ca. 53 species in the family Apocynaceae, mainly distributed in tropical and subtropical Asia and from Oceania to the Pacific coast. Among them, ca. 11 species occur in South China, Southwest China and Taiwan [1]. Some species, such as $M$. suaveolens and $M$. henyri have been used in Chinese folk medicine for the treatment of meningitis in children, rheumatic heart diseases, diuresis, bone fracture and so on [2,3]. M. scandens is used as a decongestive, against migraines, sinusitis and otitis [4]. In Australia, an aq extract of $M$. australis, when injected into a dog, produced a sharp drop in blood pressure, accompanied by an increase in the depth of respiration and decrease in rate [5]. Current crude alkaloid mixtures and purified alkaloids from some Melodinus species have demonstrated antitumor and antibacterial activities [6,7]. Many characteristic melodinus alkaloids, such as meloscine, epimeloscine, scandine and vincadifformine, had for a long time attracted great interest of synthetic organic chemists as challenging targets due to their marked diversity and complicated architectures $[8,9,10]$ And more than twenty alkaloids were reviewed from the genus Melodinus by Sevenet et al [11]. About 14 alkaloids can be classified into the melodan skeleton and its rearranged version and derived from 18, 19-didehydrotabersonine as parent compound in the plant family Apocynaceae [12]

Recently, much attention has been paid to Melodinus plants to search active melodinus alkaloids. Extensive studies of the Melodinus genus have led to the identification of several novel alkaloids. Only 22 species of the genus have been chemically investigated and provided an array of structurally interesting indole 
alkaloids, quinoline alkaloids, dimeric indole alkaloids, diterpenoids, triterpenes and others compounds over the past few decades. Some compounds show cytotoxic and anti-inflammatory activities. In this paper, we summarize phytochemistry and pharmacological activities of the Melodinus species so as to collate the existent information on this plant.

\section{PHYTOCHEMICAL CONSTITUENTS}

By the deadline of MAY 2015, phytochemical studies on this genus led to the isolation of 263 compounds. Their structures are shown below and their names, the corresponding plant sources are collected. As can be seen, indole alkaloids are the dominant constituents within this genus.

\section{Alkaloids}

Plants of the genus Melodinus (Apocynaceae) have been proven to be good sources of alkaloids. This genus has been regarded as a rich source of monoterpenoid indole alkaloids, which originated from the condensation of tryptophan with secologanin. We now list 252 alkaloids which were obtained from the genus Melodinus. The structural characters and relationships of the major Melodinus alkaloid groups are discussed.
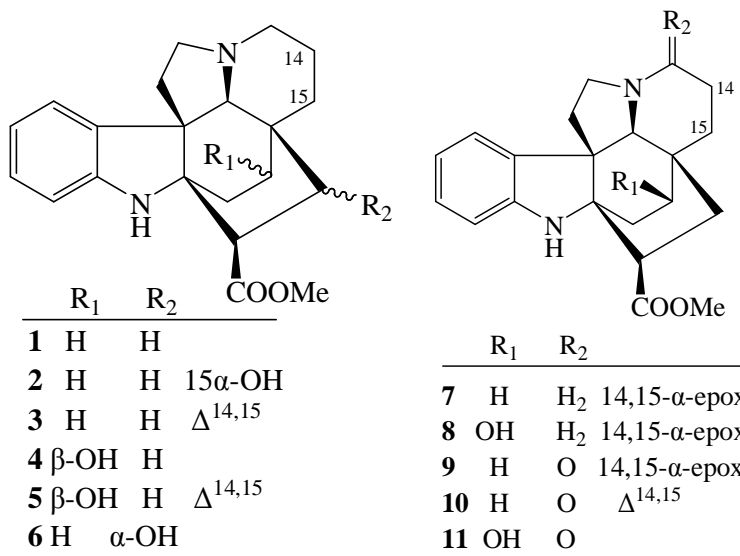

\begin{tabular}{lcll} 
& $\mathrm{R}_{1}$ & $\mathrm{R}_{2}$ & \\
\hline $\mathbf{7}$ & $\mathrm{H}$ & $\mathrm{H}_{2}$ & 14,15 - $\alpha$-epoxy \\
$\mathbf{8}$ & $\mathrm{OH}$ & $\mathrm{H}_{2}$ & 14,15 - $\alpha$-epoxy \\
$\mathbf{9}$ & $\mathrm{H}$ & $\mathrm{O}$ & $14,15-\alpha$-epoxy \\
$\mathbf{1 0}$ & $\mathrm{H}$ & $\mathrm{O}$ & $\Delta^{14,15}$ \\
$\mathbf{1 1}$ & $\mathrm{OH}$ & $\mathrm{O}$ &
\end{tabular}<smiles>CC(=O)CC1C=CC23CC(C(C)=O)C4(C1)Nc1ccccc1C42CCN3CC(C)=O</smiles>

17<smiles>CC(=O)[C@@H]1C[C@@]2(C=O)CC[C@]13Nc1ccccc1[C@]31CCN(C(C)=O)[C@H]21</smiles>

18<smiles>CC(=O)C1CC2(O)CCC34CCN5CCCC(C1)(C23)C54Nc1ccccc1</smiles>

12

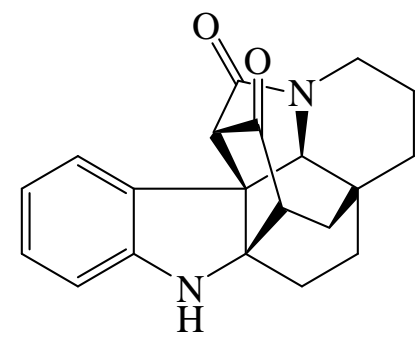

19

Figure 1: Structures of kopsinine-type alkaloids from Melodinus spps 


\begin{tabular}{|ll|}
\hline Nomenclature: & \\
A= aerial parts & $\mathrm{R}=$ root \\
$\mathrm{B}=$ stem bark & $\mathrm{S}=$ stems \\
F= fruit & $\mathrm{T}=$ twigs \\
L= leaves & $\mathrm{Tr}=$ trunk \\
$\mathrm{N}=$ not mentioned & \\
\hline
\end{tabular}

Table 1: Kopsinine-type alkaloids from genus Melodinus

\begin{tabular}{|c|c|c|c|}
\hline No. & Compound name & Species & Ref. \\
\hline 1 & Kopsinine & $\begin{array}{l}\text { M. fusiformis }(\mathrm{R} \& \mathrm{~S}), M . \text { guillauminii }(\mathrm{A} \& \mathrm{~B}), M \text {. } \\
\text { morsei }(\mathrm{N}), M \text {. reticulatus }(\mathrm{L} \& \mathrm{~S})\end{array}$ & {$[13-16]$} \\
\hline 2 & $15 \alpha$-Hydroxykopsinine & $\begin{array}{l}\text { M. fusiformis }(\mathrm{R} \& \mathrm{~S}), \text { M. guillauminii }(\mathrm{A} \& \mathrm{~B}), M . \\
\text { hemsleyanus }(\mathrm{R}), M . \text { morsei }(\mathrm{N}) \\
\text { M. australis }(\mathrm{N}), M . \text { balansae }(\mathrm{L}), M . \text { fusiformis }(\mathrm{L}, \\
\mathrm{R} \& \mathrm{~S}), M . \text { guillauminii }(\mathrm{A} \& \mathrm{~B}), M \text {. }\end{array}$ & {$[14-17]$} \\
\hline 3 & $\begin{array}{l}\text { Venalstonine }= \\
\Delta^{6}-\text { Kopsinine }\end{array}$ & $\begin{array}{l}\text { hemsleyanus }(\mathrm{A}), M \text {. insulae-pinorum }(\mathrm{A} \& \mathrm{~B}), M \text {. } \\
\text { oblongus }(\mathrm{L}), M . \text { polyadenus }(\mathrm{L} \& \mathrm{~S}), M . \\
\text { phylliraeoides }(\mathrm{L}), M \text {. reticulatus }(\mathrm{L} \& \mathrm{~S}), M . \\
\text { scandens }(\mathrm{N}), M . \text { suaveolens }(\mathrm{N}), M . \\
\text { tenuicaudatus }(\mathrm{N})\end{array}$ & $\begin{array}{l}{[13-} \\
15,18-29]\end{array}$ \\
\hline 4 & $19 \beta$-Hydroxykopsinine & M. insulae-pinorum(A \& B) & [24] \\
\hline 5 & $19 \beta$-Hydroxyvenalstonine & $\begin{array}{l}\text { M. guillauminii( } \mathrm{A} \& \mathrm{~B}), M \text {. oblongus }(\mathrm{S}), M \text {. } \\
\text { reticulatus }(\mathrm{S} \& \mathrm{~L})\end{array}$ & {$[13,14,30$} \\
\hline 6 & $17 \alpha$-Hydroxyvenalstonine & $\begin{array}{l}\text { M. tenuicaudatus }(\mathrm{N}) \\
\text { M. australis }(\mathrm{N}), M \text {. balansae }(\mathrm{L}), M . \\
\text { celastroides }(\mathrm{A}), M \text {. fusiformis }(\mathrm{L} \& \mathrm{~S}), M \text {. }\end{array}$ & [27] \\
\hline 7 & $\begin{array}{l}\text { Venalstonidine }= \\
6,7 \xi \text {-Epoxy-kopsinine }\end{array}$ & $\begin{array}{l}\text { guillauminii }(\mathrm{A} \& \mathrm{~B}), M . \text { hemsleyanus }(\mathrm{A}), M . \\
\text { insulae-pinorum }(\mathrm{A} \& \mathrm{~B}), M . \text { morsei }(\mathrm{L} \& \mathrm{~S}), M \text {. } \\
\text { polyadenus }(\mathrm{L} \& \mathrm{~S}), M . \text { phylliraeoides }(\mathrm{L}), M . \\
\text { reticulatus }(\mathrm{L} \& \mathrm{~S}), M . \text { scandens }(\mathrm{N}), M . \\
\text { yunnanensis }(\mathrm{L} \& \mathrm{~T})\end{array}$ & $\begin{array}{l}{[13,14,18-} \\
25,28,31 \\
32]\end{array}$ \\
\hline 8 & $\begin{array}{l}19-\beta- \\
\text { Hydroxyvenalstonidine }\end{array}$ & $\begin{array}{l}\text { M. insulae-pinorum(A \& B),M. reticulatus }(\mathrm{L} \& \\
\text { S) }\end{array}$ & {$[13,24]$} \\
\hline 9 & 3-Oxovenalstonidine & M. reticulatus $(\mathrm{L} \& \mathrm{~S})$ & [13] \\
\hline 10 & 3-Oxovenalstonine & M. guillauminii $(\mathrm{A} \& \mathrm{~B})$, M. reticulatus $(\mathrm{L} \& \mathrm{~S})$ & {$[13,14]$} \\
\hline 11 & 3-Oxohydroxykopsinine & M. guillauminii $(A \& B)$ & [14] \\
\hline 12 & Melodinine L & M. tenuicaudatus $(\mathrm{N}), M$. yunnanensis & {$[27,32]$} \\
\hline 13 & Refractidine & M. australis $(\mathrm{N})$ & [33] \\
\hline 14 & Pyrifoline & M. australis $(\mathrm{N})$ & [33] \\
\hline 15 & Kopsinine B & M. henryi $(\mathrm{L} \& \mathrm{~S})$ & [34] \\
\hline 16 & 12-Methoxykopsinaline & M. henryi $(\mathrm{L} \& \mathrm{~S})$ & [34] \\
\hline 17 & Melodinine Q & M. suaveolens $(\mathrm{N})$ & [29] \\
\hline 18 & 14,15-Seco-3-oxokopsinal & M. guillauminii( $\mathrm{A} \& \mathrm{~B})$ & [14] \\
\hline 19 & 10,22-Dioxokopsane & M. henryi $(\mathrm{L})$ & [35] \\
\hline
\end{tabular}

\section{Tabersonine-group}

This stereochemical series was more commonly encountered in the tabersonine group, as indicated in structures 20-55 (Table 2, Figure 2), respectively. The simple tabersonine derivatives (20-47) were identified from sixteen Melodinus species. Compounds 30- 36, in which C (14)-C (15) were epoxidized, were also isolated from $M$. aeneus, $M$. fusiformis, $M$. hemsleyanus, $M$. henryi, M. morsei, M. oblongus, $M$. suaveolens and $M$. tenuicaudatus [15,25-29,37,38,42,43]. Continuation of study on the genus Melodinus had led to the isolation of six new monoterpenoid indole alkaloids, melodinines M- S (47-52, resp.), as well as seven known tabersonine derivatives $(25,26,29,31,45,53)$ from $M$. suaveolens by Liu et al. The structure difference of 11hydroxytabersonine and melodinine A (47), melodinine $A$ was that the benzene ring $A$ was oxidized to a dienone system. Alkaloids (51 - 53) were identified as an acetonyl derivative of tabersonine by the NMR from $M$. tenuicaudatus [29]. And melotenine $A$ (55), an unprecedented skeleton with a 6/5/5/6/7 pentacyclic rearranged 
ring system, which have been derived from tabersonine, was also isolated [41].

\section{Vindolinine-type}

This type was a small group with eleven alkaloids, 56-66 (Table 3, Fig 3), which contained a $C$ (2)-C (19) bond in an aspidospermine ring from Melodinus spps. All of them expect compound $\mathbf{6 6}$ belong to the five pair of epimers and their names, the corresponding plant sources and the reference are collected in Table 3.

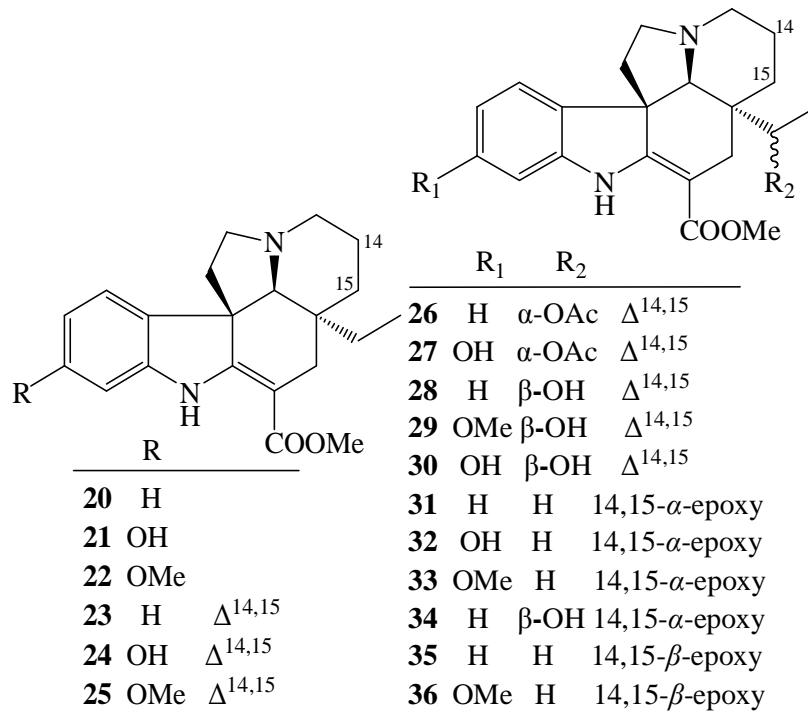

\section{Aspidospermidine-type}

Aspidospermidine alkaloids, 67-73 (Fig 4), may be derived from vincadifformine by deformylation. Eburenine (1,2-dehydroaspidospermidine) (67) and (-)-aspidospermidine (68) were isolated from air-dried leaves, twigs and aerial parts of $M$. morsei ( $\mathrm{L} \& \mathrm{~T}), M$. henryi and air-dried leaves of $M$. celastroides $[28,44,48,49]$. Previous pharmacological investigations on leaves of $M$. celastroides, epi-20, 21(+)-aspidospermidine (69), Nb-oxyepi-20, 21(+)-aspidospermidine (70), melocelinine (71) and meloceline (72) were

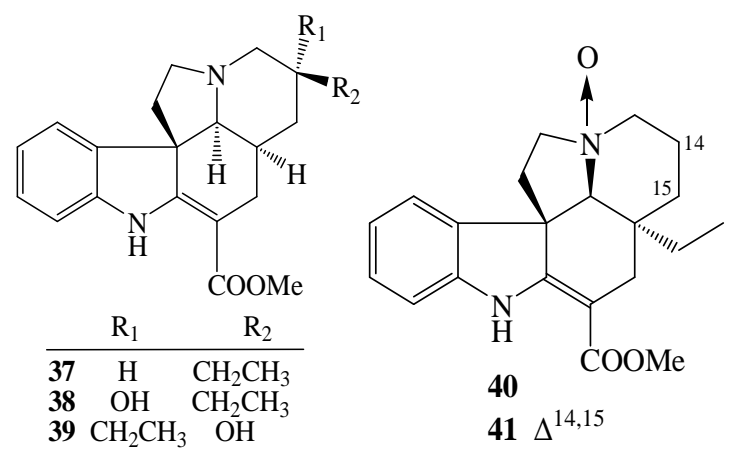

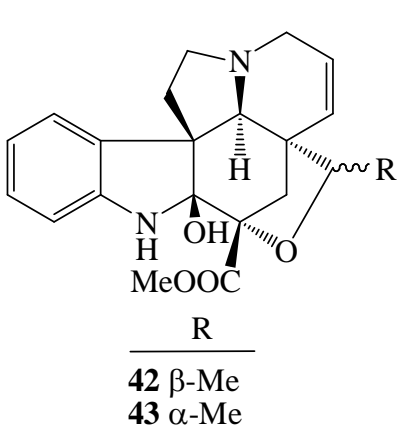

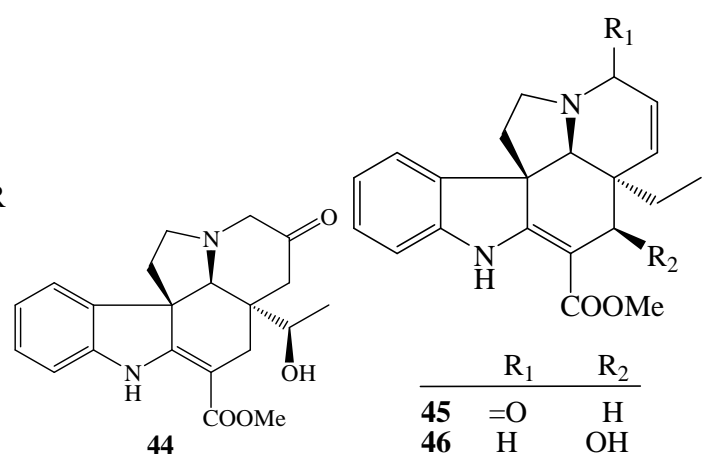

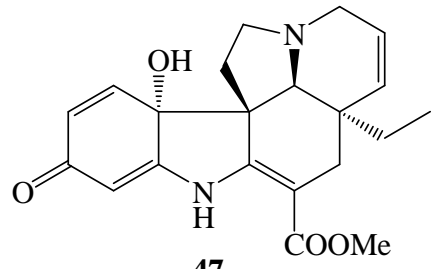<smiles>[R]c1ccc(NC2=C(C(=O)OC)C[C@]3(CC)C=CCN4CC[C@]23[C@H]4C)cc1[R]</smiles>

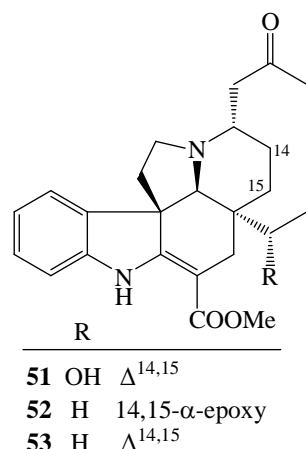

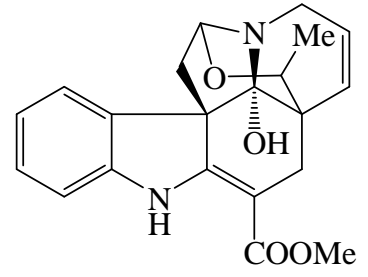

54<smiles>CC(=O)C1=C(Nc2ccccc2)C23CCN4CC=CC(C)C(C2)C4C13</smiles>

55

Figure 2: Structures of tabersonine-group alkaloids from Melodinus spps 
Table 2: Tabersonine-group alkaloids from genus Melodinus

\begin{tabular}{|c|c|c|c|}
\hline No. & Compound name & Species & Ref. \\
\hline 20 & Vincadifformine & $\begin{array}{l}\text { M. aeneus }(\mathrm{L}), M . \text { morsei }(\mathrm{L} \& \mathrm{~S})), M . \\
\text { polyadenus }(\mathrm{L} \& \mathrm{~S}), M . \text { scandens }(\mathrm{N}), M . \\
\text { suaveolens }(\mathrm{Tr})\end{array}$ & $\begin{array}{l}{[16,19,22,28} \\
, 36-38]\end{array}$ \\
\hline 21 & 11-Hydroxyvincadifformine & M. hemsleyanus $(\mathrm{A})$, M. morsei $(\mathrm{L} \& \mathrm{~S})$ & {$[25,28]$} \\
\hline 22 & 11-Methoxyvincadifformine & $\begin{array}{l}\text { M. suaveolens }(\mathrm{Tr}) \\
\text { M.aeneus }(\mathrm{L}), M . \text { balansae }(\mathrm{L}), \text { M. elastroides } \\
(\mathrm{L}), M . \text { fusiformis }(\mathrm{L}, \mathrm{R} \& \mathrm{~S}), M \text {. }\end{array}$ & [37] \\
\hline 23 & Tabersonine & $\begin{array}{l}\text { hemsleyanus }(\mathrm{A}), M . \text { henryi(F \& } \mathrm{R}), M . \\
\text { morsei }(\mathrm{L} \& \mathrm{~S}), M . \text { oblongus }(\mathrm{S}), M . \\
\text { polyadenus }(\mathrm{L} \& \mathrm{~S}), M . \text { reticulatus }(\mathrm{F}), M . \\
\text { scandens }(\mathrm{N}), M . \text { suaveolens }(\mathrm{Tr}), M . \\
\text { tenuicaudatus }(\mathrm{N}), M . \text { yunnanensis }(\mathrm{L} \& \mathrm{~T})\end{array}$ & $\begin{array}{l}{[13,15,19,20} \\
, 22,25,28- \\
32,37-42]\end{array}$ \\
\hline 24 & 11-Hydroxytabersonine & $\begin{array}{l}\text { M. axillaris }(\mathrm{R}), M \text {. balansae }(\mathrm{L}), M . \text { fusiformis }(\mathrm{R} \\
\text { \& } \mathrm{S}), M \text {. guillauminii( } \mathrm{A} \& \mathrm{~B}), M \text {. } \\
\text { hemsleyanus }(\mathrm{A} \& \mathrm{R}), M . \text { morsei }(\mathrm{L} \& \mathrm{~S}), M \text {. } \\
\text { oblongus }(\mathrm{L}), M . \text { tenuicaudatus }(\mathrm{B}) \text {, } \\
\text { M. suaveolens }(\mathrm{Tr}, \mathrm{L} \& \mathrm{~T})\end{array}$ & $\begin{array}{l}{[14,15,17,20} \\
, 25- \\
29,37,43- \\
46]\end{array}$ \\
\hline 25 & 11-Methoxytabersonine & $\begin{array}{l}\text { M. aeneus }(\mathrm{L}), M . \text { fusiformis }(\mathrm{R} \& \mathrm{~S}), M . \\
\text { guillauminii }(\mathrm{A} \& \mathrm{~B}), M . \text { hemsleyanus }(\mathrm{A}) \text {, } \\
\text { M. henryi }(\mathrm{F} \& \mathrm{R}), M . \text { polyadenus }(\mathrm{L} \& \mathrm{~S}) \text {, } \\
\text { M. reticulatus }(\mathrm{F}), M . \text { suaveolens }(\mathrm{Tr}), M \text {. } \\
\text { tenuicaudatus }(\mathrm{B}), M . \text { yunnanensis }(\mathrm{L} \& \mathrm{~T})\end{array}$ & $\begin{array}{l}{[13-} \\
15,22,25,27 \\
29,32,37,38 \\
42,43]\end{array}$ \\
\hline 26 & 19-Acetyltabersonine & M. morsei(L \& S),M. suaveolens $(\mathrm{N})$ & {$[28,29]$} \\
\hline 27 & $\begin{array}{l}\text { 11-Hydroxy-19- } \\
\text { acetyltabersonine }\end{array}$ & M. tenuicaudatus $(\mathrm{N}), M$. morsei( $\mathrm{L} \& \mathrm{~S})$ & {$[27,28]$} \\
\hline 28 & 19R-Hydroxytabersonine & M. suaveolens(Tr) & [37] \\
\hline 29 & $\begin{array}{l}\text { 11-Methoxy-19R- } \\
\text { hydroxytabersonine }\end{array}$ & M. suaveolens $(\mathrm{Tr})$ & {$[29,37]$} \\
\hline 30 & 11,19R-Dihydroxytabersonine & $\begin{array}{l}\text { M. fusiformis }(\mathrm{R} \& \mathrm{~S}), \text { M. hemsleyanus }(\mathrm{A}) \text {, } \\
\text { M. suaveolens }(\mathrm{Tr})\end{array}$ & {$[15,25,37]$} \\
\hline 31 & Lochnericine & $\begin{array}{l}\text { M. aeneus }(\mathrm{L}), M . \text { morsei }(\mathrm{L} \& \mathrm{~S}), M \text {. } \\
\text { suaveolens }(\mathrm{N}), M . \text { yunnanensis }(\mathrm{L} \& \mathrm{~T})\end{array}$ & $\begin{array}{l}{[28,29,3} \\
2,38]\end{array}$ \\
\hline 32 & $\begin{array}{l}\text { 11-Hydroxy-14,15 } \alpha \text { - } \\
\text { Epoxytabersonine }\end{array}$ & $\begin{array}{l}\text { M. fusiformis }(\mathrm{R} \& \mathrm{~S}), M . \\
\text { hemsleyanus }(\mathrm{A}), \\
\text { M. morsei }(\mathrm{L} \& \mathrm{~S}), \text { M. tenuicaudatus }(\mathrm{N})\end{array}$ & $\begin{array}{l}{[15,25,2} \\
7,28]\end{array}$ \\
\hline $\begin{array}{l}33 \\
34\end{array}$ & $\begin{array}{l}\text { Hazuntine } \\
\text { Cathovalinine }\end{array}$ & $\begin{array}{l}\text { M. suaveolens }(\mathrm{Tr}), \text { M. tenuicaudatus }(\mathrm{B}) \\
\text { M. suaveolens }(\mathrm{Tr})\end{array}$ & $\begin{array}{l}{[37,43]} \\
{[37]}\end{array}$ \\
\hline 35 & $\beta$-Epoxytaberonine & M. oblongus $(\mathrm{L})$ & [26] \\
\hline 36 & Lochnerinine & M. aeneus $(\mathrm{L}), M$. henryi( $\mathrm{F} \& \mathrm{R})$ & {$[38,42]$} \\
\hline 37 & (+)-20-R-Pseudovincadifformine & M. polyadenus $(\mathrm{L} \& \mathrm{~S})$ & [22] \\
\hline 38 & (+)-20-R-Pandoline & M. polyadenus $(\mathrm{L} \& \mathrm{~S})$ & [22] \\
\hline 39 & (+)-20-S-Pandoline & M. polyadenus $(\mathrm{L} \& \mathrm{~S})$ & {$[22]$} \\
\hline 40 & Vincadifformine $\mathrm{N}_{b}$-oxide & M. morsei( $\mathrm{L} \& \mathrm{~S})$, M. yunnanensis $(\mathrm{L} \& \mathrm{~T})$ & {$[28,32]$} \\
\hline 41 & Tabersonine $\mathrm{N}_{4}$-oxide & M. yunnanensis $(L \& \mathrm{~T})$ & [32] \\
\hline 42 & Melobaline & M. balansae $(\mathrm{L})$ & [20] \\
\hline 43 & Vincoline & $\begin{array}{l}\text { M. hemsleyanus }(\mathrm{R}), \text { M. morsei(A), } \\
\text { M. suaveolens }(\mathrm{Tr})\end{array}$ & {$[17,36,37,44]$} \\
\hline 44 & Baloxine & M. balansae $(\mathrm{L})$ & [20] \\
\hline 45 & 3-Oxotabersonine & M. suaveolens, $M$. yunnanensis $(\mathrm{L} \& \mathrm{~T})$ & {$[29,32]$} \\
\hline 46 & $\begin{array}{l}\text { 17-Hydroxy-14,15- } \\
\text { dihydrotabersonine }\end{array}$ & M. yunnanensis $(\mathrm{L} \& \mathrm{~T})$ & [32] \\
\hline 47 & Melodinine M & M. suaveolens $(\mathrm{N})$ & [29] \\
\hline 48 & Melodinine $\mathrm{N}$ & M. suaveolens $(\mathrm{N})$ & {$[29]$} \\
\hline 49 & Melodinine $\mathrm{O}$ & M. suaveolens $(\mathrm{N})$ & [29] \\
\hline 50 & Melodinine P & M. suaveolens $(\mathrm{N})$ & [29] \\
\hline 51 & Melodinine R & M. suaveolens(N) & [29] \\
\hline 52 & Melodinine S & M. suaveolens $(\mathrm{N})$ & [29] \\
\hline 53 & $3 \alpha$-Acetonyltabersonine & M. suaveolens $(\mathrm{N})$ & [29] \\
\hline 54 & Buxomeline & M. celastroides $(\mathrm{L})$ & [40] \\
\hline 55 & Melotenine A & M. tenuicaudatus $(\mathrm{N})$ & {$[41]$} \\
\hline
\end{tabular}




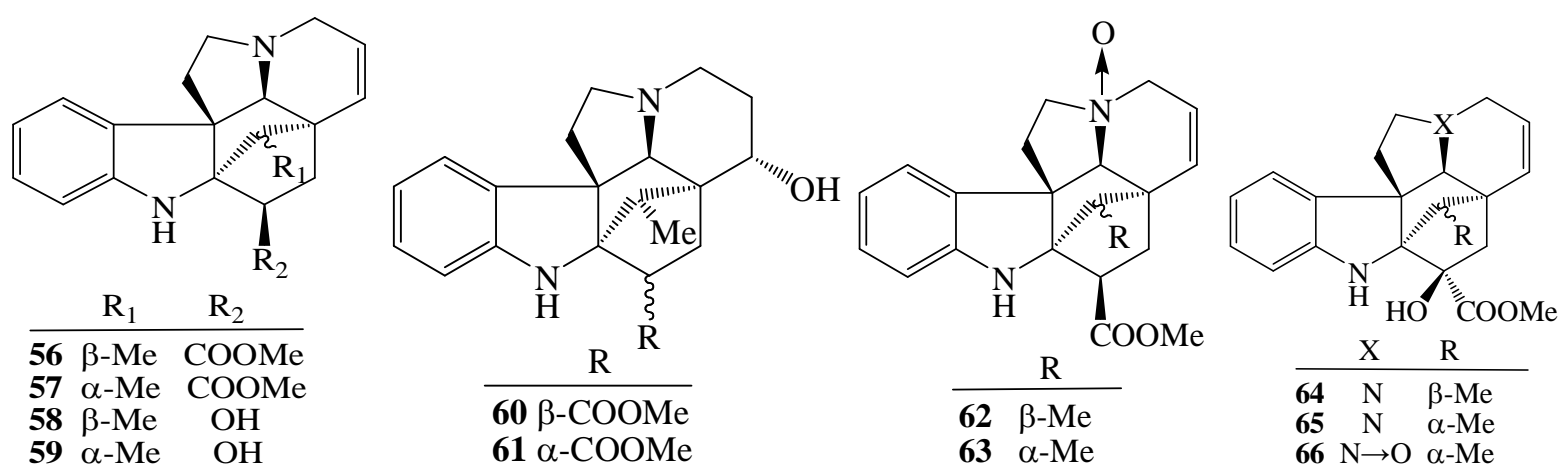

Figure 3: Structures of vindolinine-type alkaloids from Melodinus spps

Table 3: Vindolinine-type alkaloids from genus Melodinus

\begin{tabular}{|c|c|c|c|}
\hline No. & Compound name & Species & Ref \\
\hline 56 & $\begin{array}{l}\text { 19S-Vindolinine } \\
=\text { Vindolinine }\end{array}$ & $\begin{array}{l}\text { M. balansae }(\mathrm{L}), M . \text { fusiformis }(\mathrm{R} \& \mathrm{~S}) \text {, } \\
\text { M. hemsleyanus, } M . \text { morsei( } \mathrm{N},), \\
\text { M. Oblongus }(\mathrm{S}), M . \text { hylliraeoides }(\mathrm{L}) \text {, } \\
\text { M. Suaveolens }(\mathrm{Tr}), M . \text { tenuicaudatus }(\mathrm{B})\end{array}$ & $\begin{array}{l}{[15,16,20} \\
23,27,28,3 \\
0,37,43]\end{array}$ \\
\hline 57 & $\begin{array}{l}\text { 19R-Vindolinine } \\
=\text { Epivindoline }\end{array}$ & $\begin{array}{l}\text { M. celastroides }(\mathrm{L}), M . \text { hemsleyanus }(\mathrm{A}) \text {, } \\
\text { M. morsei }(\mathrm{L} \& \mathrm{~S}), \text { M. phylliraeoides }(\mathrm{L}) \text {, } \\
\text { M. oblongus }(\mathrm{S})\end{array}$ & $\begin{array}{l}{[16,23,25} \\
28,30,40]\end{array}$ \\
\hline $\begin{array}{l}58 \\
59\end{array}$ & \multirow{2}{*}{$\begin{array}{l}\text { 16-Hidroxyvindolinine } \\
\text { 16-Hidroxyepivindolinine } \\
\text { 15 } \alpha \text {-Hydroxy-14,15- } \\
\text { dihydrovindolinine }\end{array}$} & $\begin{array}{l}\text { M. oblongus(S) } \\
\text { M. oblongus(S) }\end{array}$ & $\begin{array}{l}{[30]} \\
{[30]}\end{array}$ \\
\hline 60 & & M. Morsei(N) & {$[16,47]$} \\
\hline 61 & $\begin{array}{l}\text { 15a-Hydroxy-14,15-dihydro- } \\
\text { 16-epivindolinine }\end{array}$ & M. morsei(N) & {$[16,47]$} \\
\hline 62 & Vindolinine $\mathrm{N}_{b}$-oxide & $\begin{array}{l}\text { M. balansae }(\mathrm{L}), \text { M. hemsleyanus }(\mathrm{R}) \text {, } \\
\text { M. morsei }(\mathrm{N}), M . \text { phylliraeoides }(\mathrm{L}) \text {, } \\
\text { M. tenuicaudatus }(\mathrm{B})\end{array}$ & $\begin{array}{l}{[16,17,20} \\
23,43]\end{array}$ \\
\hline 63 & Epivindolinine $\mathrm{N}_{b}$-oxide & $\begin{array}{l}\text { M. balansae }(\mathrm{L}), M . \text { morsei }(\mathrm{N}), M \text {. } \\
\text { phylliraeoides }(\mathrm{L}), \text { M. tenuicaudatus }(\mathrm{B})\end{array}$ & $\begin{array}{l}{[16,20,23,} \\
43]\end{array}$ \\
\hline $\begin{array}{l}64 \\
65\end{array}$ & \multirow{2}{*}{$\begin{array}{l}\text { 16 } \beta \text {-Hydroxy-19R-vindolinine } \\
16 \beta \text {-Hydroxy-19S-vindolinine } \\
16 \beta \text {-Hydroxy-19S-vindolinine } \\
\text { N-oxide }\end{array}$} & $\begin{array}{l}\text { M. hemsleyanus }(\mathrm{R} \& \mathrm{~A}) \\
\text { M. hemsleyanus }(\mathrm{A})\end{array}$ & $\begin{array}{l}{[17,25]} \\
{[25]}\end{array}$ \\
\hline 66 & & M. hemsleyanus $(\mathrm{R})$ & [17] \\
\hline
\end{tabular}

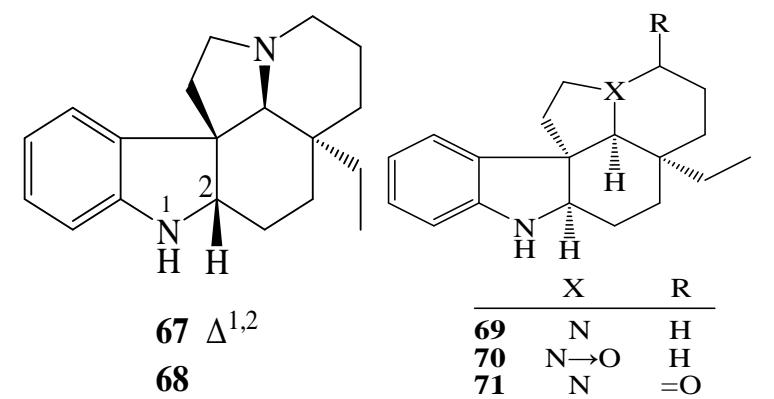

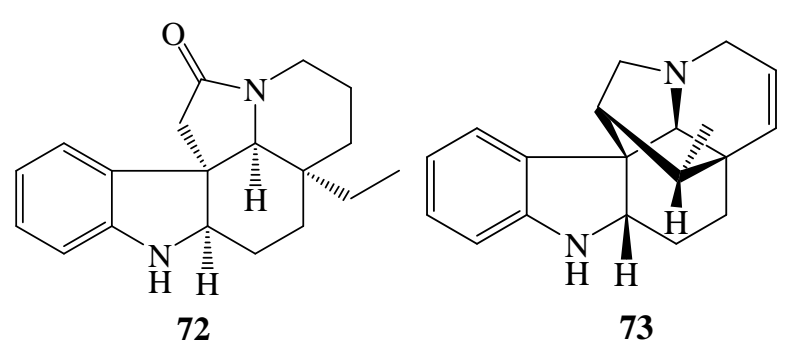

Figure 4: Structures of aspidospermidine-type alkaloids from Melodinus spps

isolated [50]. 6,7-Didehydroisotuboxenine (73) was only aspidospermidine alkaloid isolated from leaves and twigs of $M$. yunnanensis [32].

\section{Aspidospermidine-type}

Aspidospermidine alkaloids, 67-73 (Fig 4), may be derived from vincadifformine by deformylation.
Eburenine (1,2-dehydroaspidospermidine) (67) and (-)-aspidospermidine (68) were isolated from air-dried leaves, twigs and aerial parts of $M$. morsei $(L \& T), M$. henryi and air-dried leaves of $M$. celastroides $[28,44,48,49]$. Previous pharmacological investigations on leaves of $M$. celastroides, epi-20, 21(+)-aspidospermidine (69), Nb-oxyepi-20, 21(+)-aspidospermidine (70), 
melocelinine (71) and meloceline (72) were isolated [50]. 6,7-Didehydroisotuboxenine (73) was only aspidospermidine alkaloid isolated from leaves and twigs of M. yunnanensis [32].

\section{Quebrachamine derivatives}

The group is exemplified by quebrachamine (74) and its derivatives (75-85) (Fig 5), which lack the $\mathrm{C}(7)-\mathrm{C}(21)$ bond of aspidospermidine from $M$. australis, $M$. axillaries, $M$. fusiformis, $M$. morsei and $M$. suaveolens $[16,18,28,37,44,45]$. In this group, (S)-quebrachamine (74) and (+)-17methoxy- quebrachamine (75), rhazidine (76), 11-hydroxyrhazidine (77) and axillarisinine (78) were isolated from roots of $M$. australis $[18,45]$. The leaves and twigs of $M$. yunnanensis were collected in Honghe (PR China), provided four new quebrachamine derivatives, $14 \beta, 15 \beta$-20Squebrachamine (80), 3-oxo-voaphylline (81), $2 \alpha, 7 \alpha$-dihydroxy-dihydrovoaphylline (82), 16ß,21ß-epoxy-vincadine (83), and three known compounds (+)-voaphylline (79), voalenine (84) and voaphylline hydroxyindolenine (85) [32]. Suaveolenine (86) was that a structure with a lactam ring and an additional five-membered ring containing an oxygen atom had been found from the trunk of $M$. suaveolens [37].
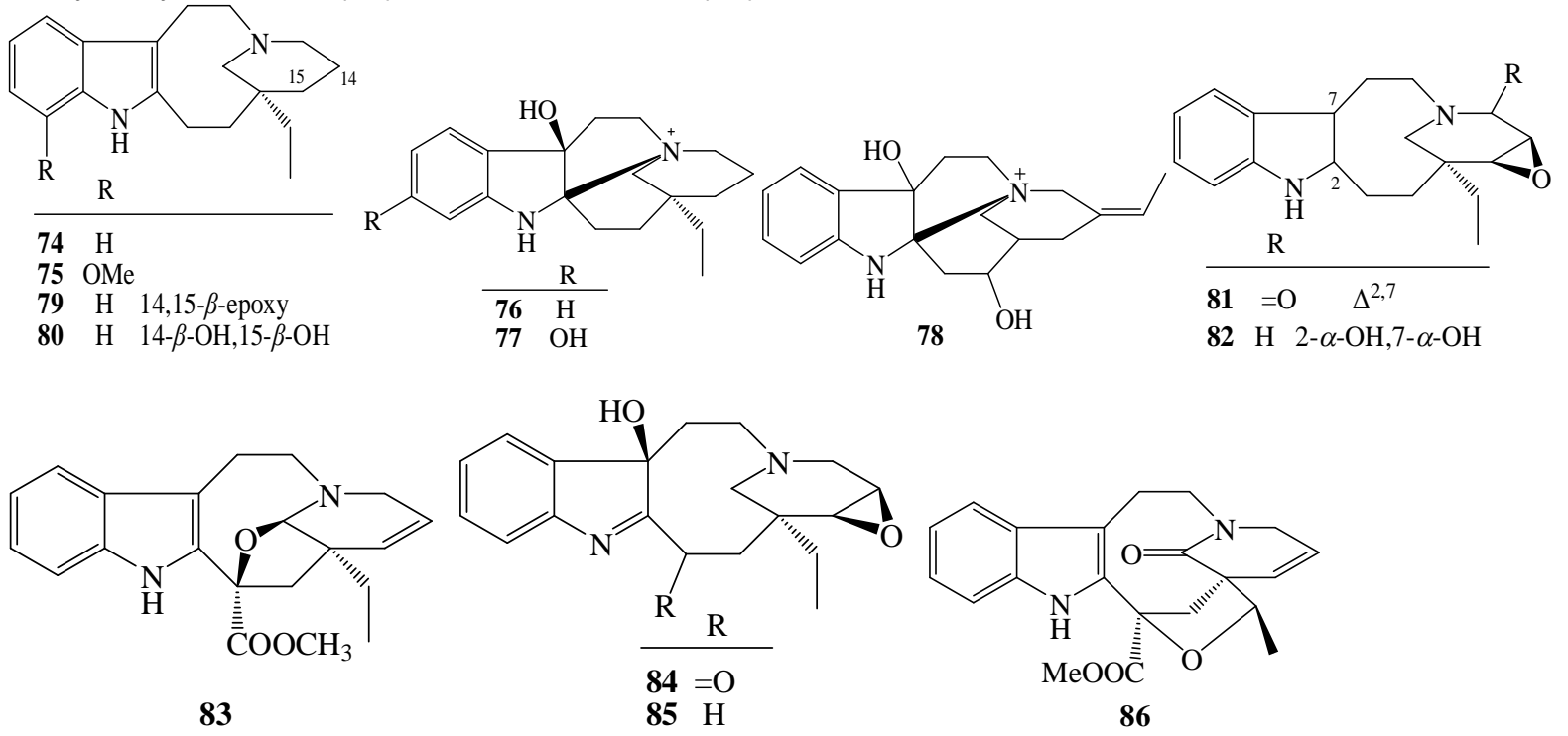

Figure 5: Structures of quebrachamine derivatives alkaloids from Melodinus spps

\section{Eburnamine-type, 87- 114 (Table 4. Fig 6)}

Five pair of epimers, 87- 96, were isolated from nine Melodinus species. Four eburnamine-type alkaloids including two new ones, melodinines F$\mathrm{G}(\mathbf{9 7}, 98)$, together with O-methylepivincanol (100), (-)-eburnamenine (101) were isolated from $M$. henryi $[35,48]$. $\Delta 14$-Isoeburnamine (105) and (+)-isoeburnamine (106) were obtained from $M$. celastroides, $M$. henryi, $M$. oblongus and $M$. henryi $[30,40,42,52]$. Five indole alkaloids, $\Delta 14$ vincamenine N4-oxide (99), meloyunine (109), 14ß-hydroxymeloyunine (110) and its epimer (111), and 16,19-epoxy- $\Delta 14$-vincanol (113) were described from M. yunnanensis [32].

Tubotaiwine-type (Figure 7)

Tubotaiwine (114) and tubotaiwine $\mathrm{N}$-oxide (115) were isolated from $M$. aeneus, $M$. axillaris, $M$. fusiformis, $M$. hemsleyanus, $M$. oblongus, $M$. suaveolens [15,17,26,38,45,55]. From leaves and twigs of $M$. yunnanensis and air-dried of $M$. henryi yielded seven metabolites, 19Shydroxytubotaiwine (116) and its N4-oxide (117), 19R-methoxytubotaiwine (118) and its 19Rdemethylation derivative (119), kopsiyunnanine F3 (120), melodinine D (121) and 20-hydroxytubotaiwine (122) [32,48]. ( \pm )-Condylocarpine

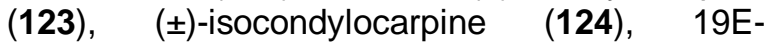
isocondylocarpine N4-oxide (125) and 19,20dihydrocondylocapine (126) were obtained from $M$. australis $(\mathrm{R}), M$. henryi $(\mathrm{L} \& \mathrm{R}), M$. khasianus (B), M. oblongus (S), M. yunnanensis ( $\mathrm{L} \& \mathrm{~T}$ ) $[18,26,32,35,42,51]$.

\section{Akuammicine-type (Figure 8)}

Four akuammicines derivatives, akuammicine (127) and its $\mathrm{N}$-oxide (128), norfluorocurarine (129) and its $\mathrm{N}$-oxide (130), stricticine (131) and compactinervine (132), were isolated from $M$. axillaries (R), M. henryi $(\mathrm{L})$, M. oblongus $(\mathrm{S})$ and 
Table 4: Eburnamine-type and derivatives alkaloids from genus Melodinus

\begin{tabular}{|c|c|c|c|}
\hline No. & Compound name & Species & Ref. \\
\hline 87 & $O$-methyl- $\Delta^{14}$-vincanol & M. tenuicaudatus $(\mathrm{N})$, M. yunnanensis $(\mathrm{L} \& \mathrm{~T})$ & {$[27,32]$} \\
\hline 88 & 16-Epi-O-methyl- $\Delta^{14}$-vincanol & M. khasianus(B), M. yunnanensis $(\mathrm{L} \& \mathrm{~T})$ & {$[32,51]$} \\
\hline & & M. celastroides $(\mathrm{L}), M$. henryi(F \& R), $M$. & {$[24,30,32$,} \\
\hline 89 & $\Delta^{14}$-Vincanol $=\Delta^{14}$-Eburnamine & $\begin{array}{l}\text { Insulae- pinorum(B \& A), } \\
\text { tenuicaudatus }(\mathrm{B}), M . \text { yunnanensis }(\mathrm{L} \& \mathrm{~T})\end{array}$ & $\begin{array}{l}39,42,43 \\
49,51]\end{array}$ \\
\hline 90 & 16-Epi- $\Delta^{14}$-vincanol & $\begin{array}{l}\text { M. celastroides }(\mathrm{L}), M \text {. insulae-pinorum(B \& } \\
\text { A), M. oblongus }(\mathrm{S})\end{array}$ & {$[24,30,49]$} \\
\hline 91 & $\begin{array}{l}\Delta^{14} \text {-Vincine }=14,15- \\
\text { Dehydrovincamine }\end{array}$ & $\begin{array}{l}\text { M. henryi }(\mathrm{F} \& \mathrm{R}), \text { M. polyadenus }(\mathrm{L} \& \mathrm{~S}), M \text {. } \\
\text { suaveolens }(\mathrm{Tr}), M . \text { tenuicaudatus }(\mathrm{B}), M \text {. } \\
\text { yunnanensis }(\mathrm{L} \& \mathrm{~T})\end{array}$ & $\begin{array}{l}{[2,22,32,3} \\
7,43]\end{array}$ \\
\hline 92 & Epi-16-dehydro-14,15-vincine & M. $\operatorname{aeneus}(\mathrm{L})$ & [38] \\
\hline 93 & $\Delta^{14}$-Vincamine & M. henryi $(\mathrm{F} \& \mathrm{R})$, M. yunnanensis $(\mathrm{L} \& \mathrm{~T})$ & {$[32,42,52]$} \\
\hline 94 & $\begin{array}{l}\text { Epi-16- } \Delta^{14} \text {-vincamine } \\
(+)-16-\text { epi- } \Delta^{14} \text {-vincamine }\end{array}$ & $\begin{array}{l}\text { M. aeneus }(\mathrm{L}), M . \text { henryi }(\mathrm{R}) \text {, } \\
\text { M. yunnanensis }(\mathrm{L} \& \mathrm{~T})\end{array}$ & {$[32,38,52]$} \\
\hline 95 & $\begin{array}{l}\text { 14-Epieburnamine = } \\
\text { (-)-Isoeburnamine; }(3 \alpha, 14 \beta, 16 \alpha)\end{array}$ & M. henryi $(\mathrm{L})$ & {$[35,48]$} \\
\hline $\begin{array}{l}96 \\
97\end{array}$ & $\begin{array}{l}(+) \text {-Eburnamine }=(+) \text {-Vincanol } \\
\text { Melodinine F }\end{array}$ & $\begin{array}{l}\text { M. celastroides }(\mathrm{L}), \text { M. henryi }(\mathrm{L}) \\
\text { M. henryi }(\mathrm{N})\end{array}$ & $\begin{array}{l}{[35,39]} \\
{[48]}\end{array}$ \\
\hline 98 & Melodinine G & M. henryi $(\mathrm{N})$ & [48] \\
\hline 99 & $\Delta^{14}$-Vincamenine $\mathrm{N}_{4}$-oxide & M. yunnanensis $(\mathrm{L} \& \mathrm{~T})$ & [32] \\
\hline 100 & O-Methylepivincanol & M. henryi $(\mathrm{N})$ & [48] \\
\hline 101 & (-)-Eburnamenine = Vincamenine & M. henryi $(\mathrm{L})$ & {$[35,48]$} \\
\hline 102 & 11-Methoxy- $\Delta^{14}$-vincamenine & M. guillauminii $(B$ \& A) & [14] \\
\hline 103 & 11-Methoxy- $\Delta^{14}$-vincanol & M. guillauminii(B \& A) & [14] \\
\hline 104 & $\Delta^{14}$-Vincamenine & M. yunnanensis $(\mathrm{L} \& \mathrm{~T})$ & [53] \\
\hline 105 & $\Delta^{14}$-Isoeburnamine & $\begin{array}{l}\text { M. celastroides }(\mathrm{L}) \text {, } \\
\text { M. henryi }(\mathrm{F} \& \mathrm{R}), M \text {. oblongus }(\mathrm{S})\end{array}$ & {$[30,40,42]$} \\
\hline 106 & (+)-Isoeburnamine & M. henryi $(\mathrm{R})$ & [52] \\
\hline 107 & 20-Oxo-eburnamine & M. henryi(L\& S) & [34] \\
\hline 108 & 19-Oxoeburnamine & M. henryi (L\& S) & [34] \\
\hline 109 & Meloyunine & M. yunnanensis $(\mathrm{L} \& \mathrm{~T})$ & [32] \\
\hline 110 & $14 \beta$-Hydroxymeloyunine & M. yunnanensis $(L \&$ T) & [32] \\
\hline 111 & 16-Decarbomethoxy-vincapusine & M. yunnanensis $(L \& \mathrm{~T})$ & [32] \\
\hline 112 & 16,19-Epoxy- $\Delta^{14}$-vincanol & M. yunnanensis $(L \&$ T $)$ & [32] \\
\hline 113 & 14,17-Epoxy-eburnamine & M. henryi $(\mathrm{F}, \mathrm{R} \& \mathrm{~S})$ & [54] \\
\hline
\end{tabular}
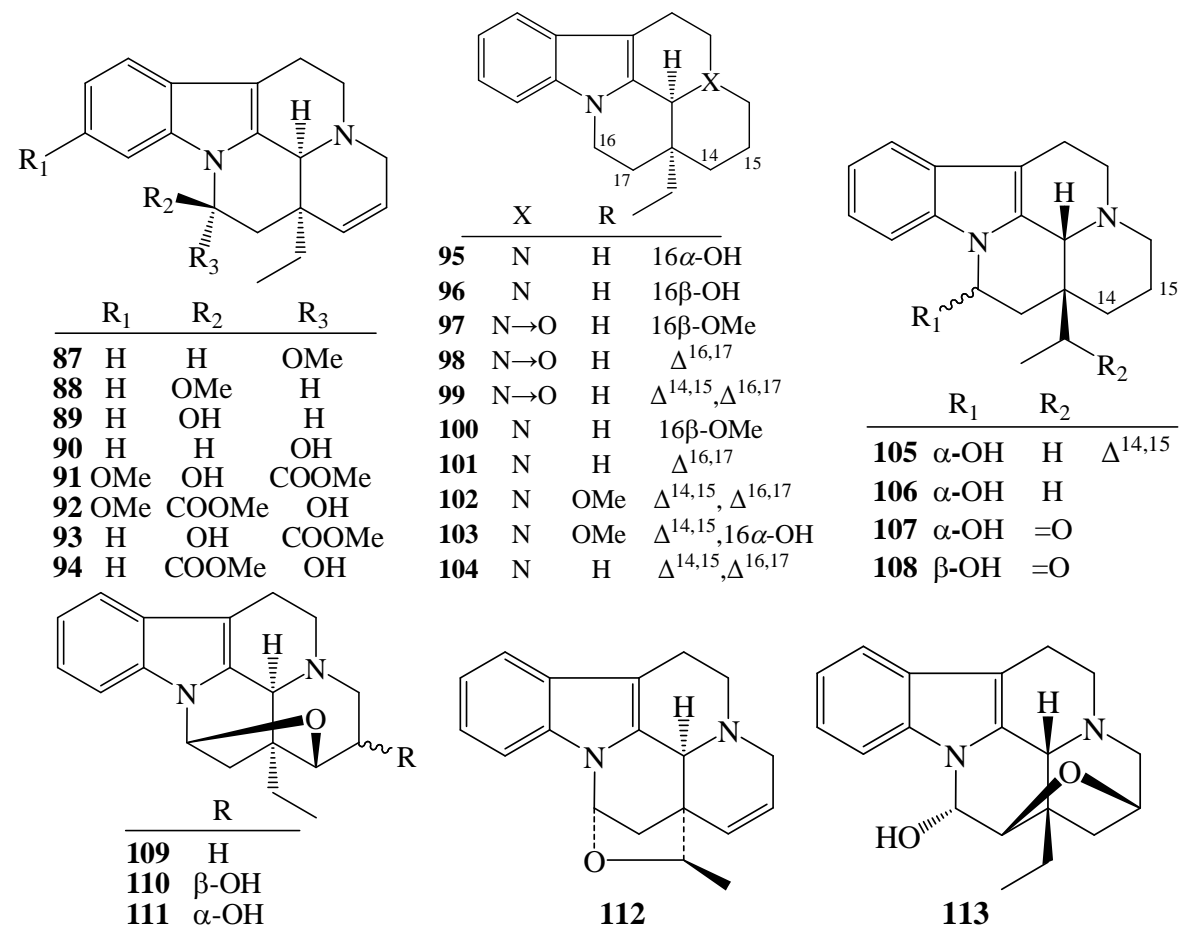

$106 \alpha-\mathrm{OH} \quad \mathrm{H}$

$107 \alpha-\mathrm{OH}=\mathrm{O}$

$108 \beta-\mathrm{OH}=\mathrm{O}$

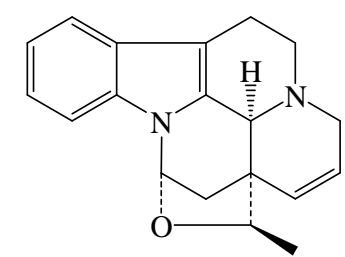

112

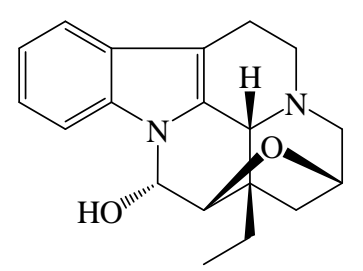

113

Figure 6: Structures of eburnamine-type alkaloids from Melodinus spps 
M. tenuicaudatus, $M$. suaveolens $(L \& T)$ and $M$. yunnanensis (L \& T) [26,32,35,43,45,46].

Geissoschizine-type and derivatives (Table 5(a) and (b), Figure 9)

Twenty-five constituents, 133-157, were isolated from $M$. acutiflorus, $M$. australis, $M$. fusiformis, $M$. hemsleyanus, $M$. khasianus, $M$. oblongus and $M$. tenuicaudatus

$[15,20,27,28,32,43,50,54]$
Picraline-type (133-139) and anhydrojmalinetype alkaloids (140-141) are biogenetically considered to be derived from geissoschizine (144) with its derivative by ring closure between the $C(7)$ and $C(16)$ positions and between the $C(5)$ and $C(16)$. As a common intermediate, geissoschizine (144) would also provide the mavacurine-type alkaloids (149-150) and tetrahidroalstonin (151).

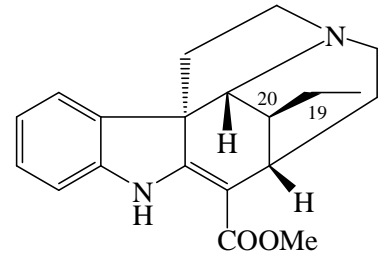

$12019,20-\beta$-epoxy $12119,20-\alpha$-epoxy $12220-\alpha-\mathrm{OH}$

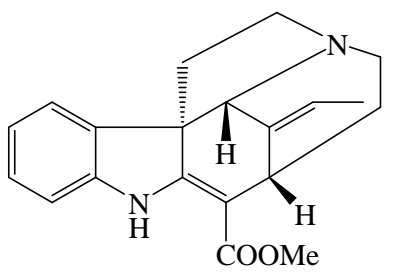

123

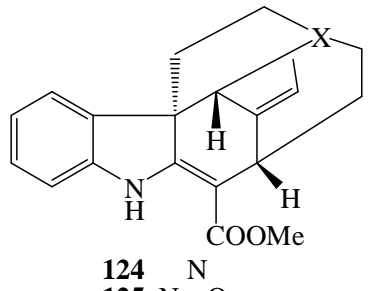

$125 \mathrm{~N} \rightarrow \mathrm{O}$<smiles>COC(=O)C1=C2Nc3ccccc3[C@]2(C)[C@H]2CN3CC[C@H]1[C@H]23</smiles>

Figure 7: Structures of tubotaiwine -type alkaloids from Melodinus spps

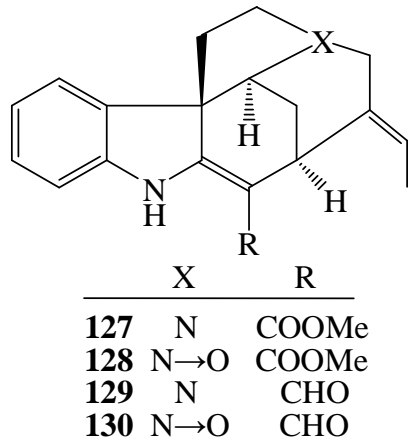

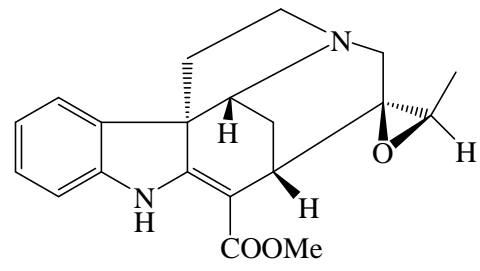

131<smiles>CC(=O)C1=C2Nc3ccccc3[C@]23CCN2C[C@](O)(C(C)O)C[C@]1(C2)C3</smiles>

132

Figure 8: Structures of akuammicine-type alkaloids from Melodinus spps

\section{Leuconotis alkaloid 376 and its analogues (Figure 10)}

Melodinines A-C (158-160, resp.), together with leuconotis alkaloid 376 (161), possessed 22 skeletal carbons arranged compactly in six rings, were isolated from $M$. henryi by RP-18 gel and Sephadex LH-20 [48].

Axillarisine (162) was found from dry root powder of $M$. axillaris by Yan et al [45]. Three other novel alkaloids, melohenine A (163), an unusual C24 monoterpenoid indole alkaloid with additional skeletal carbons arranged compactly in eight rings, and henrycinols $A-B$ (164-165) with an a cinnamoyl group system were also isolated from roots of $M$. henryi $[52,57]$. The relative configuration of henrycinols $A$ and $B$ was determined by NOESY analysis.

Trop J Pharm Res, December 2015; 14(12): 2333 
Table 5: Geissoschizine-type and derivatives alkaloids from genus Melodinus

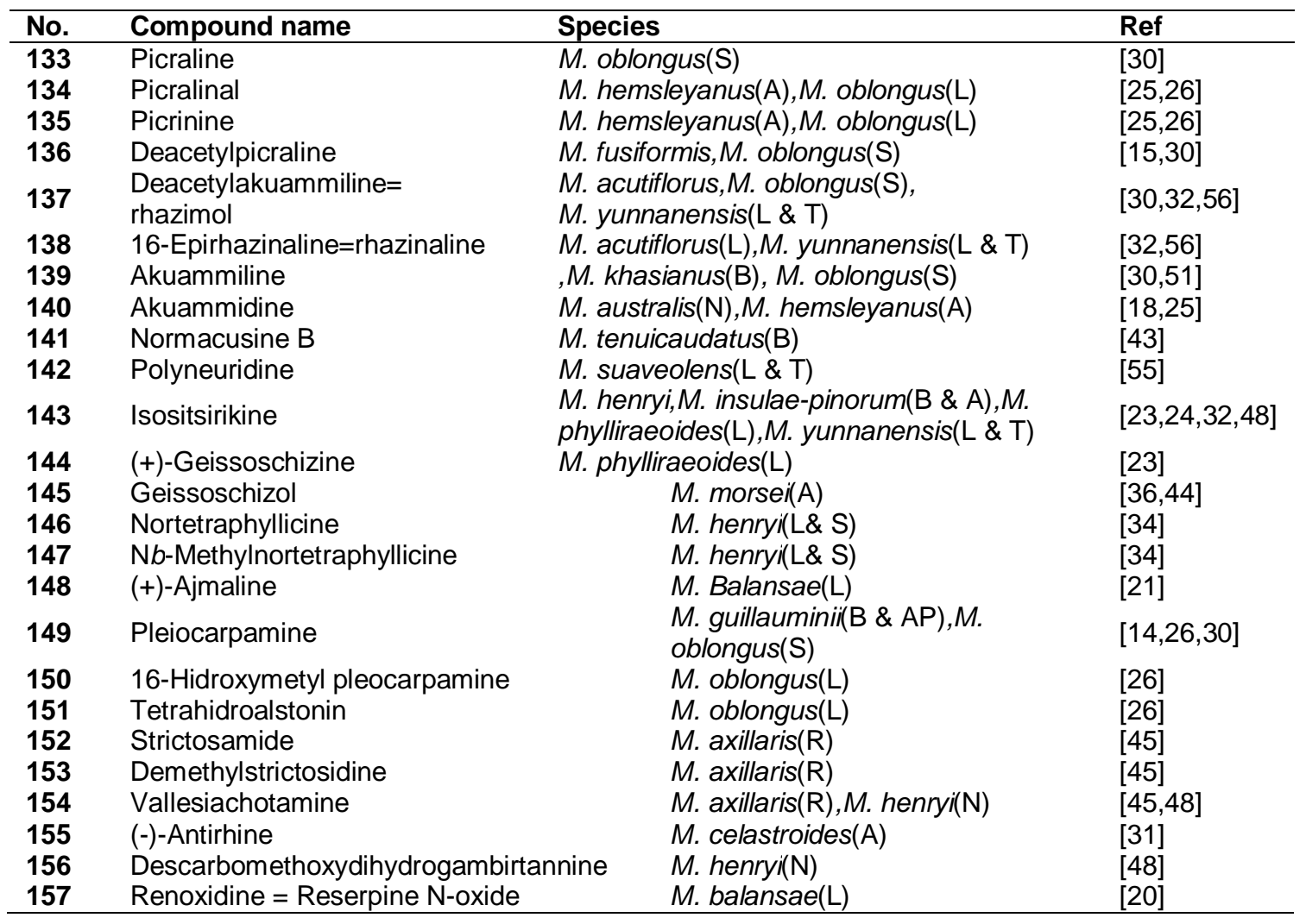

\section{Other indole alkaloids, 166-178 (Figure 11)}

Two known compounds, stemmadenine (166) and its $\mathrm{N}$-oxide (167), were isolated from $M$. henryi $[18,35,48]$. N-acyl-indolinique (168) and a new diazaspiroindole alkaloid, melodinine $E$ (169) were structural type seldom reported from M. morsei and M. henryi, resp. [28,48]. 6/7-Seco rearranged spiro-indolone alkaloids, meloyunines $A(\mathbf{1 7 0})$ and $B(\mathbf{1 7 1})$ were elucidated from leaves and twigs of $M$. yunnanensis. These structures were elucidated based on NMR, FTIR, UV, and MS spectroscopic data [53]. Mehri et al reported melonine (172) and Nb-oxy-melonine (173) from $M$. celastroides possibly artifacts due to the use of $\mathrm{CH}_{2} \mathrm{Cl}_{2}$ or $\mathrm{HCl}$ as an extraction solvent $[40,49]$. In 1978, Baassou et al found (-)-ibogamine (174) from $M$. aeneus [38]. Two novel alkaloids arbophylline (175) and 15- $\beta$-methoxy- 14 , 15dihydroandranginine (176) were isolated from leaves and twigs of $M$. yunnanensis [32]. Melodinoxanine (177), which derived from an oxygenated derivative of isocarapanaubine (178), was a unique oxindole alkaloid with an extra oxygen atom in the C-ring of a heteroyohimbine skeleton from the stems and leaves of $M$. henryi growing in Yunnan, China [34].

\section{Monoterpenoid quinoline alkaloids}

\section{Scandine derivatives, 179-183 (Figure 12)}

In 1969, Bernauer et al identified scandine (179), which was a naturally occurring biologically active product derived from plants, was important as a possible starting material for the syntheses of valuable pharmaceuticals, from $M$. scandens first time [58]. Then, this compound and 10hydroxyscandine (180) were isolated from the other seven plants of $M$. fusiformis, $M$. hemsleyanus, $M$. henryi, $M$. khasianus, $M$. oblongus, $M$. tenuicaudatus, $M$. scandens, $M$. suaveolens $\quad[2,15,17,19,25,27,28,29,30,43,46$, 55,59]. And the absolute configuration has aroused the great concern [60]. The crystal structure and absolute configuration of (+)scandine have been determined by X-ray diffraction $[61,62]$. Some fo scandine derivatives, 10-methoxyscandine (181), scandine $\mathrm{Nb}$-oxide (182), melodinine $U$ (183) and $T$ (184), melodinhenine C-D (185-186) obtained from $M$. tenuicaudatus, $M$. fusiformis, $M$. henryi, $M$. suaveolens $[15,27,29,55,63]$. The interesting chemical significance of the Melodinus plants prompted researchers to initiate a phytochemical study on the twigs and leaves of $M$. suaveolens, which led to the isolation of two new quinoline alkaloids, 14, 15-dihydroscandine (187) and 15ß- 

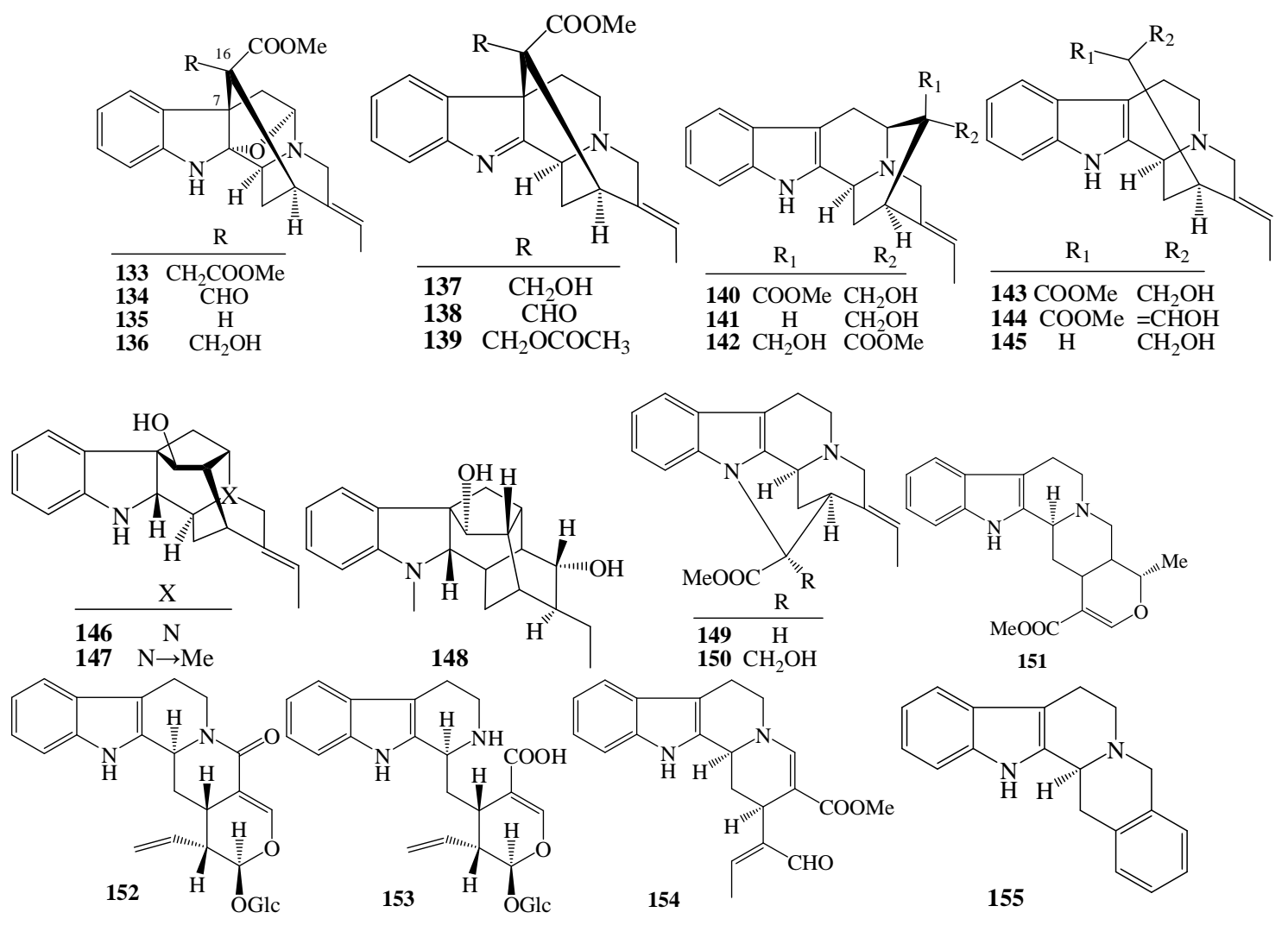<smiles>CC[C@H](CO)[C@H]1CCN2CCc3c([nH]c4ccccc34)[C@H]2C1</smiles>

156

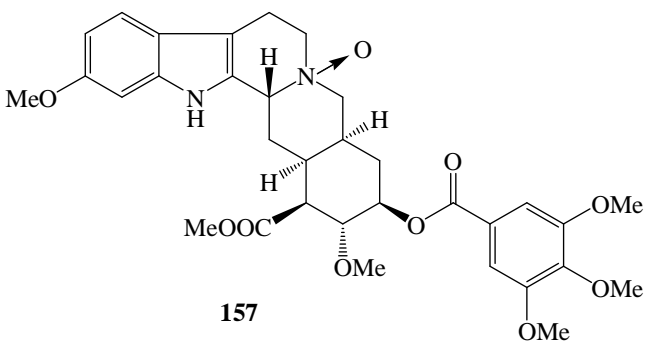

Figure 9: Structures of geissoschizine-type and derivatives alkaloids from Melodinus spps

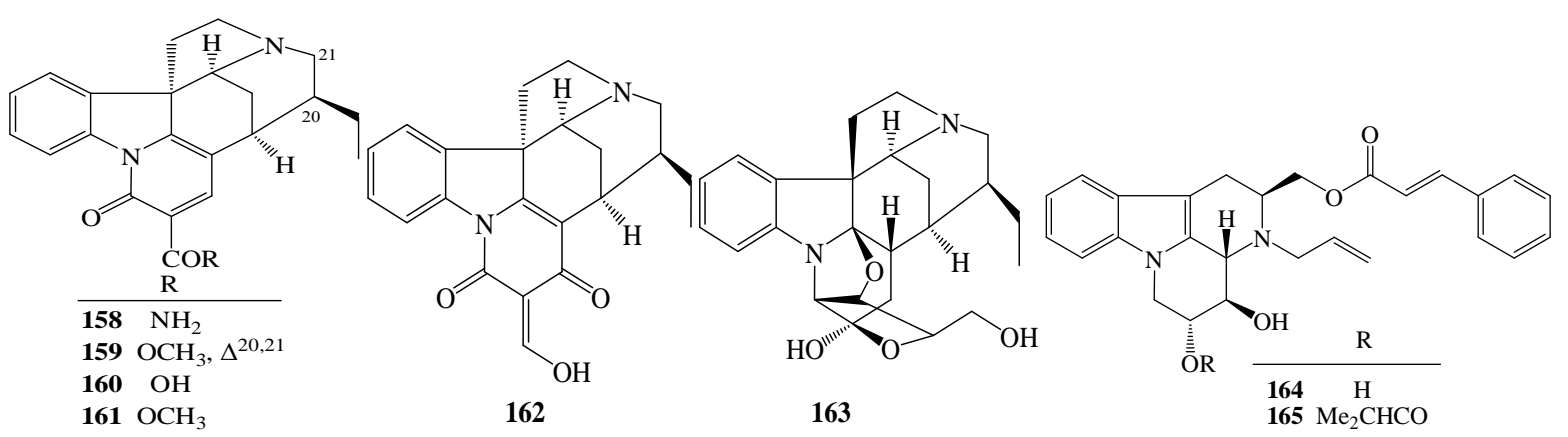

Figure 10: Structures of leuconotis alkaloid 376 and its analogues alkaloids from Melodinus spps 

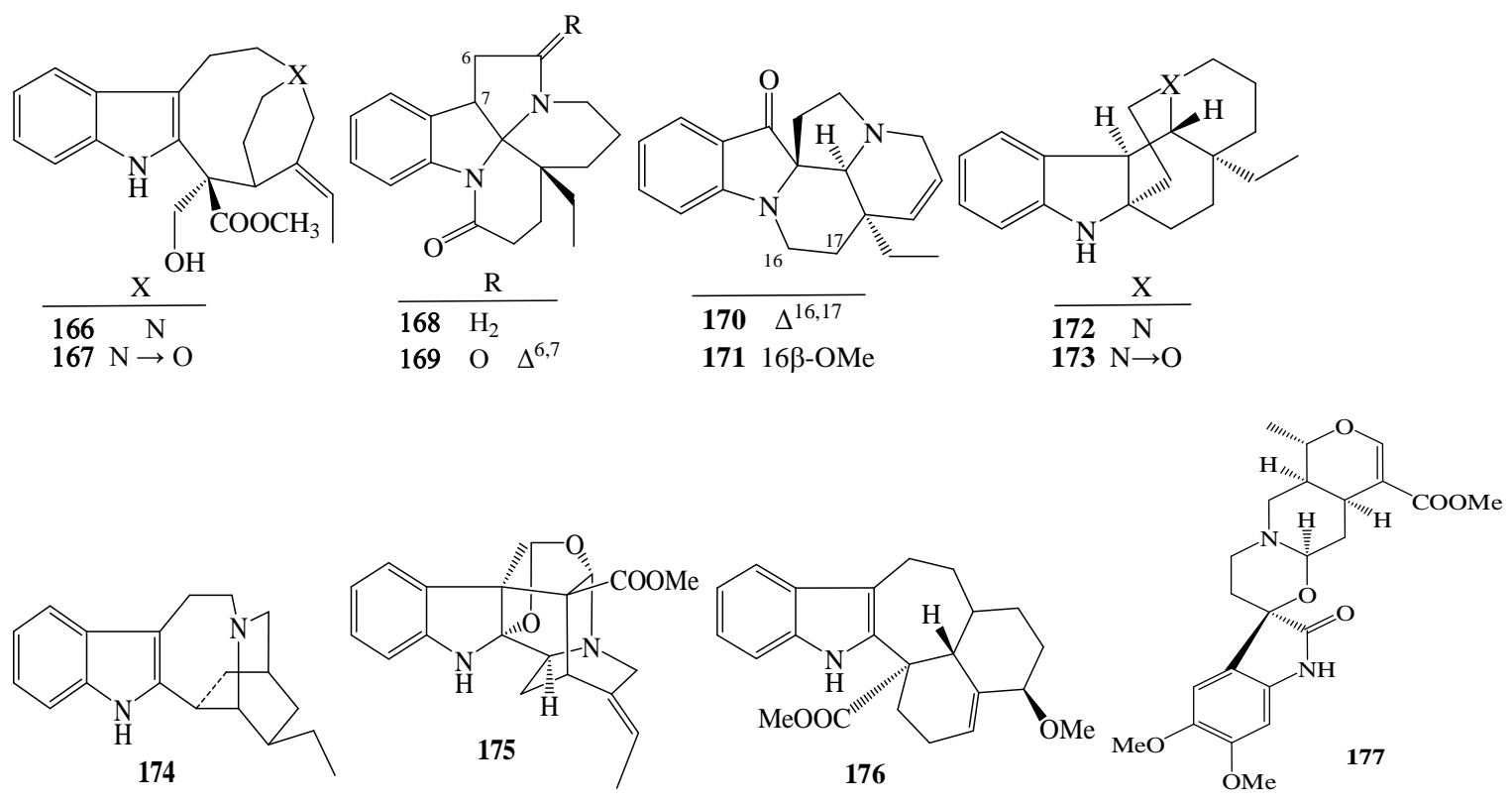<smiles>COc1cc2c(cc1OC)[C@]1(CCN3C[C@H]4[C@H](C[C@H]31)C(C(C)=O)=CO[C@@H]4C)C(=O)N2</smiles>

Figure 11: Structures of other indole alkaloids from Melodinus spps

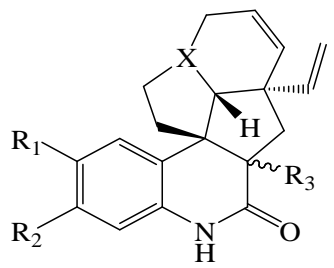

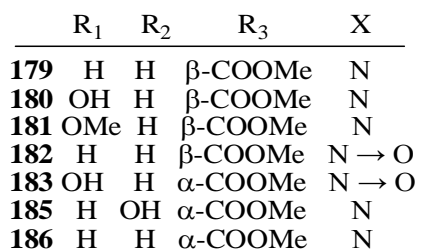<smiles>CC(=O)C12CC(C(C)=O)(C(C)=O)C34CC=CCN3CCC14c1ccccc1NC2=O</smiles>

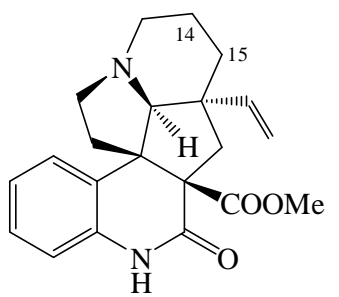

187

$18815-\beta-\mathrm{OH}$

189 14,15- $\beta$-epoxy

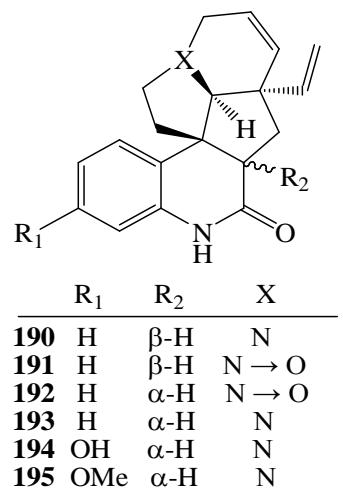

(N)

\begin{tabular}{rrr}
$R_{1}$ & $R_{2}$ & $R_{3}$ \\
\hline $196 \mathrm{H}$ & $\mathrm{Me}$ & $\alpha-\mathrm{H}$
\end{tabular}

$197 \mathrm{Me} \quad \mathrm{H} \quad \alpha-\mathrm{H}$

$198 \mathrm{H} \quad \mathrm{Me} \quad \beta-\mathrm{H}$

$199 \mathrm{Me} \quad \mathrm{H} \quad \beta-\mathrm{H}$

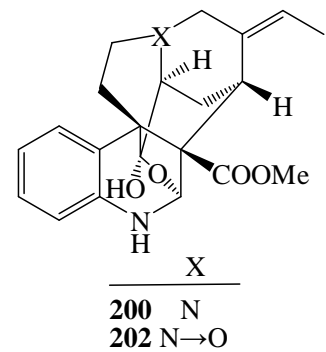

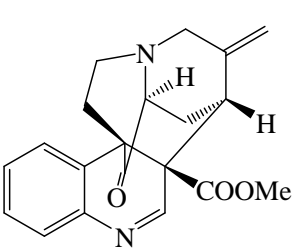

201<smiles>CC[C@]12C=CCN3Cc4c(n(c5ccccc45)C1=O)[C@@]32CO</smiles>

203

Figure 12: Structures of scandine derivatives quinoline alkaloids from Melodinus spps 
hydroxy- 14,15-dihydroscandine (188), along with a known alkaloids 14,15 - $\beta$-epoxyscandine (189) $[46,55]$.

\section{Meloscine derivatives, 190-195 (Figure 12)}

Meloscine (190) and its $\mathrm{Nb}$-oxide (191) were isolated from $M$. hemsleyanus, $M$. oblongus, M.scandens, M. suaveolens [25,30,55,59]. Four epi-meloscine compounds, $\mathrm{N}$-oxyepimeloscine (192), epimeloscine (193), 9hydroxyepimeloscine (194) and 9-methoxy epimeloscine (195), were isolated from $M$. scandens [19,58,60,61,64]. Meloscandonine (196) was isolated from $M$. fusiformis, $M$. hemsleyanus, $M$. khasianus, $M$. scandens, $M$. oblongus, $\quad M$. tenuicaudatus $[15,19,25-$ 28,55,58,61]. And the structure of stereochemistry by UV, IR, NMR and X-ray [65,66]. 19-Epimeloscandonine (197) and melodinhenine E-F $(198,199)$ obtained from $M$. fusiformis, $M$. hemsleyanus, $M$. henryi and $M$. oblongus $[25,28,63]$. On the structure elucidation of the main alkaloids of $M$. acutiflorus, which led to a revision of the structures of rhazicine (200) and rhazimine (201), were first isolated. Further investigation of the extract of leaves of $M$. acutiflorus gave a new alkaloid, rhazicine $\mathrm{N}$ oxide $(202)[56,67]$. To our knowledge, the first report of the co-occurrence of monoterpenoid indoles meloyunine $C(\mathbf{2 0 3})$ and precursor $\Delta 14$ vincamenine (104) in same plant, supporting the biosynthesis of quinoline from $M$. yunnanensis and concurring with previous in vivo tracer experiments in the literature [53].

Other monomeric alkaloids, 204-210 (Figure 13)

(-)-Rhazinilam (204) was a natural compound isolated from $M$. australis and $M$. henryi, whose tetracyclic structure possesses an axially chiral phenyl-pyrrole subunit bridged by a ninemembered lactam ring $[18,35,48,68]$. Melohenine B (205), 14, 15-dehydromelohenine B (206) and
14-O-ethyl-substituted $(3 \alpha, 14 \alpha, 16 \alpha)-2,7-$ secoeburnamine derivative (207) with an unprecedented 6/9/6/6 tetracyclic ring system, regarded as a key intermediate from indole to quinoline alkaloids were obtained from $M$. henryi and $M$. yunnanensis $[35,52,55]$. Three simple alkaloids, venoterpine (208), ( $\square$ )-R/S-1-(3pyridyl)ethanol (209) and 2, 5-diphenyloxazole (210), were also isolated from $M$. aeneus, $M$. axillaris and $M$. celastroides $[33,38,45]$.

\section{Bisindole alkaloids}

\section{Eburnamine derivative dimeric alkaloids (Figure 14)}

Fifteen bisindole alkaloids, melonines C-D (211-212), having an eburnamine-aspidospermidine linkage, guillauminiine (213), paucivenine (214), tenuicausine (215), demethylteuicausine (216) and melodinine $\mathrm{J}$ (217), possessing an eburnamine-tabersonine skeleton, melodinine $\mathrm{H}-\mathrm{I}$ (218-219), melodinhenine A-B (220-221) of the eburnaminevindolinine type, and melonine A (222), melaxillarinine (223) and melaxillaridine (224) with an eburnamine-quebrachamine derivatives, were isolated from eight Melodinus species by column chromatography (CC),TLC, RP-18 gel, Sephadex $\quad$ LH-20 $\quad[6,14,21,27,42,43,45,65,68$ 71].The absolute configuration of 220 and 221 was determined using ECD exciton chirality method. $M$. insulaepinorum furnished an eburnamine-kopsinine bisalkaloid, insulopinine (225) [24]. From M. celastroides, celastromeline (226) and celastromelidine (227) were the tertiary analogues of Le chlorure de chloromethyl celastromelinium (228) and Le chlorure de chloromethyl celastromelidinium (229), and their quasi-dimeric structures were not likely to be artifacts [49]. The laster eburnaminediazaspiroindole bisindole alkaloids, melonine $B$ (230) was isolated as a pale-red powder from $M$. henryi [71].<smiles>CC[C@]12CCCn3ccc(c31)-c1ccccc1NC(=O)CC2</smiles>

204

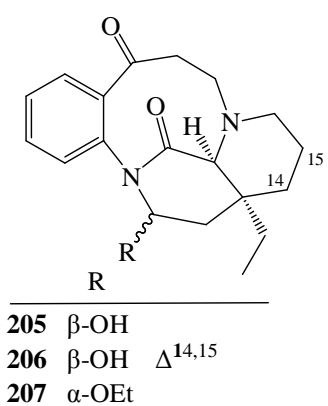

$207 \alpha$-OEt<smiles>C[C@@H]1c2cnccc2C[C@H]1O</smiles>

208<smiles>CC(O)c1cccnc1</smiles>

209

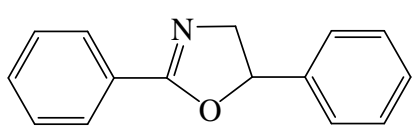

210

Figure 13: Structures of other monomeric alkaloids alkaloids from Melodinus spps 

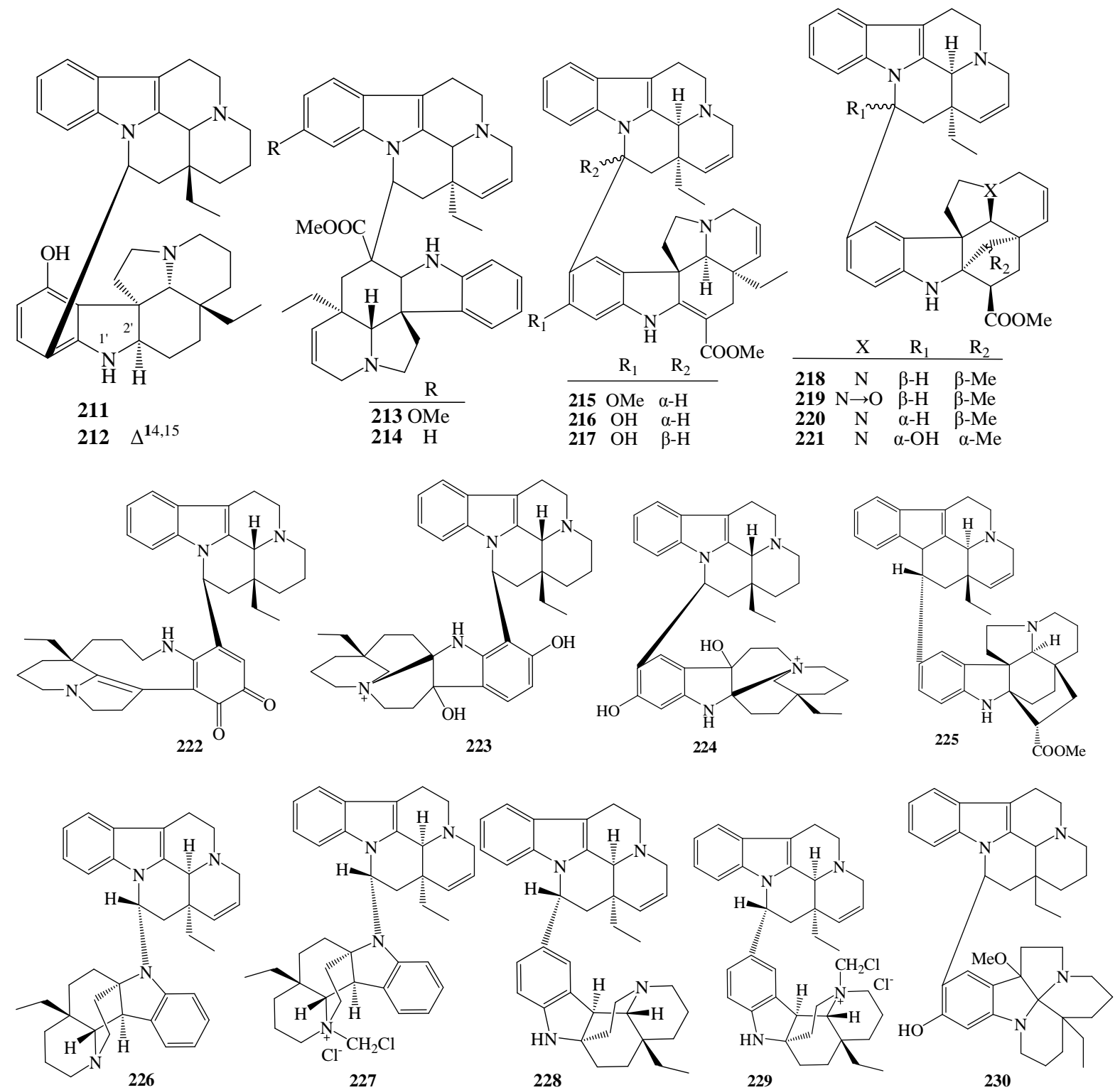

Figure 14: Structures of eburnamine-type dimeric alkaloids from Melodinus spps

Tabersonine derivative dimeric alkaloids, 231247 (Figure 15)

In this part, we list all tabersonine derivative dimeric alkaloids except eburnamine-tabersonine type. Tabersonine-tabersonine skeleton, melodinine $K$ (231) and melomorsine I (232) were isolated from $M$. tenuicaudatus and $M$. fusiformis, resp. [27,28]. In 2013, melosuavine D$\mathrm{F}$ (233-235) were identified by Liu et al from twigs and leaves of $M$. suaveolens [69]. From $M$. suaveolens and $M$. scandens, three tabersoninescandine, melosuavine A-C (236-238), and three tabersonine-vindolinine type, melosuavine G-H (239-240) and scandomelidine (241) were obtained $[69,72]$. The isolation and structural determination of lochnericine-kopsinine derivatives from M. yunnanensis and M. morsei, meloyine (242) and melornorsine (243), were studied $[32,44]$. Four 18, 19-dehydrotabersoninvincadifformine derivatives, scandomelonine (244) and its epimer (245), scandomeline (246) and its epimer (247), were isolated from $M$. scandens $[72,73]$.

Other dimeric alkaloids (248-252), (Figure 15)

Melaxilline (248) and melaxillinine (249) were obtained from $M$. axillaris [45]. To investigate antitumor indole alkaloids from the leaves and twigs of M. morsei, melofusine I (250) was subjected to RP-18 silica gel CC on eluting with $70 \%-75 \%$ aq. $\mathrm{MeOH}$ [28]. M. celastroides contained two other dimeric alkaloids, Dichlorure de methylene $\mathrm{Nb}, \mathrm{Nb}^{\prime}$ [bis-[(+)-meloninium] (251) and methylene 10,10'-bis-[(+)-Na-norvallesami- 

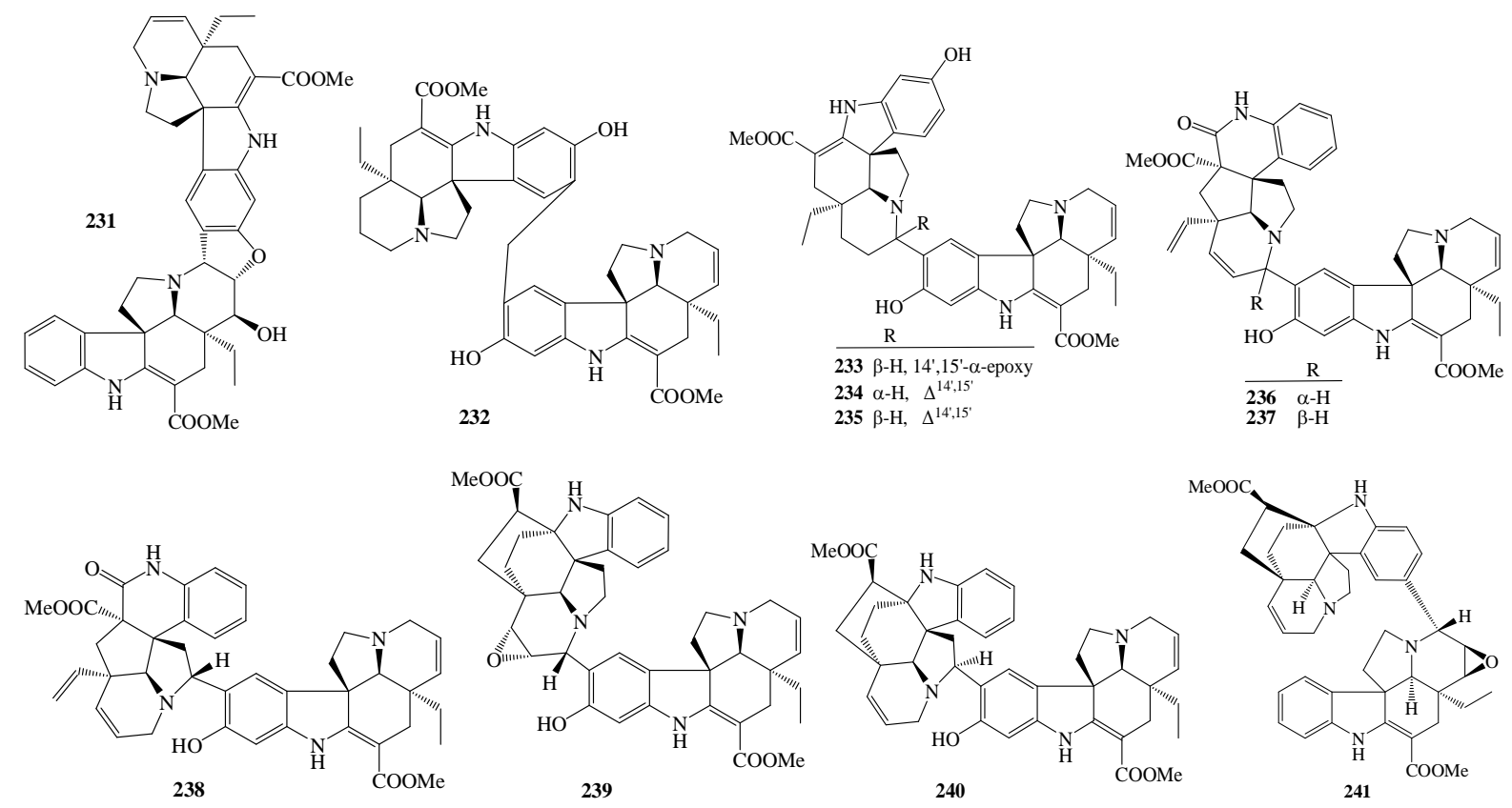

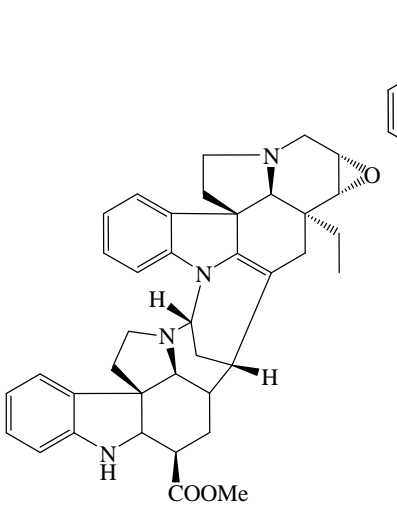

242

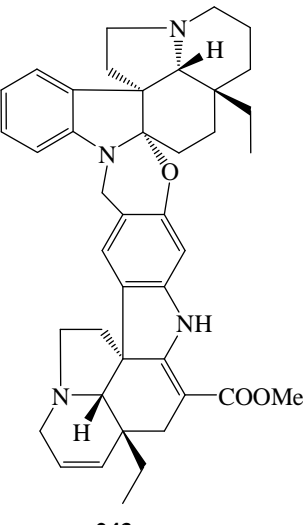

243
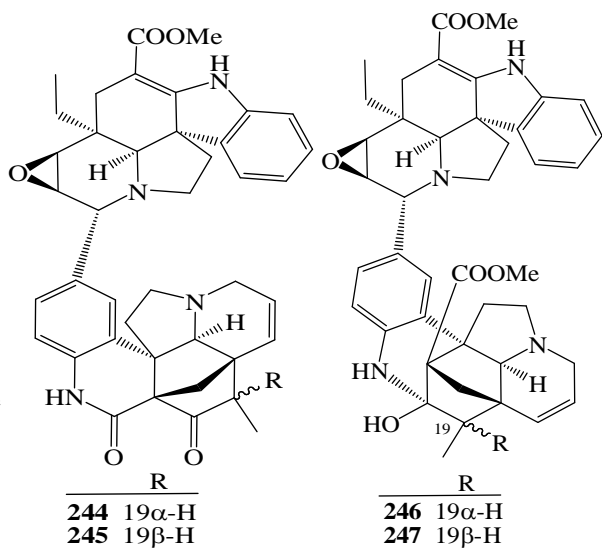

Figure 15: Structures of eburnamine-type dimeric alkaloids from Melodinus spps

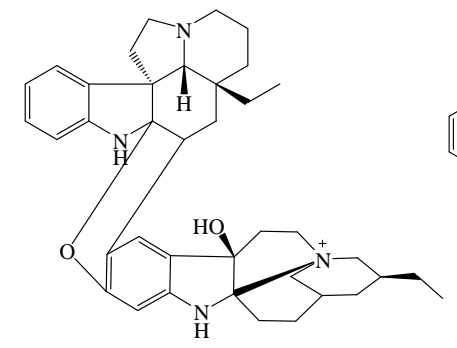

248

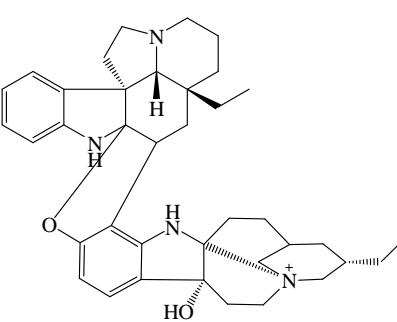

249

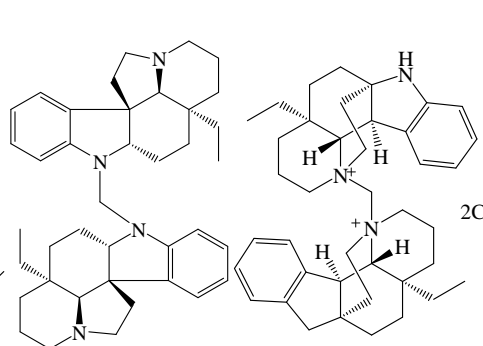

251

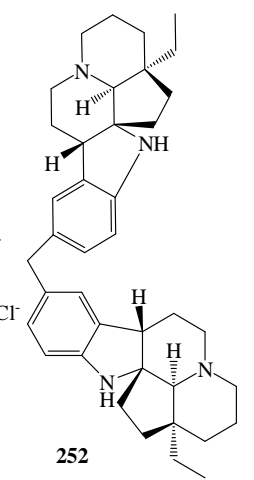

Figure 16: Structures of other dimeric alkaloids from Melodinus spps 


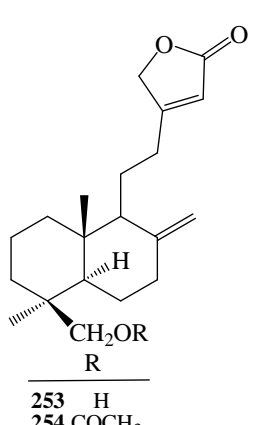

$254 \mathrm{COCH}_{3}$

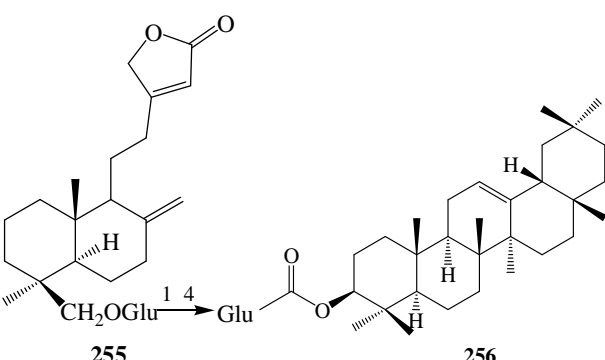

256
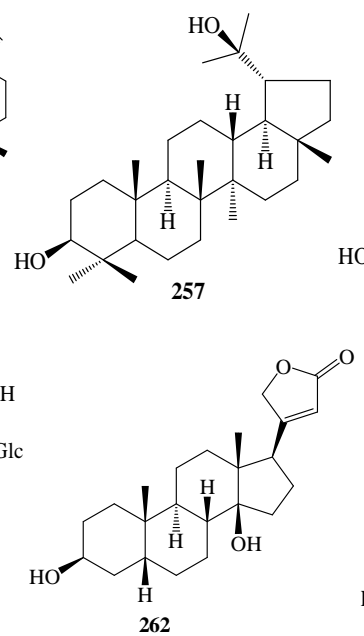

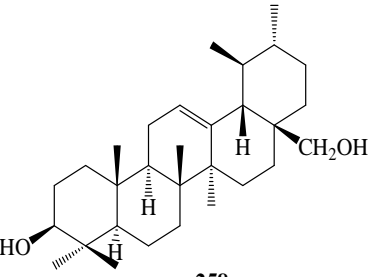

258
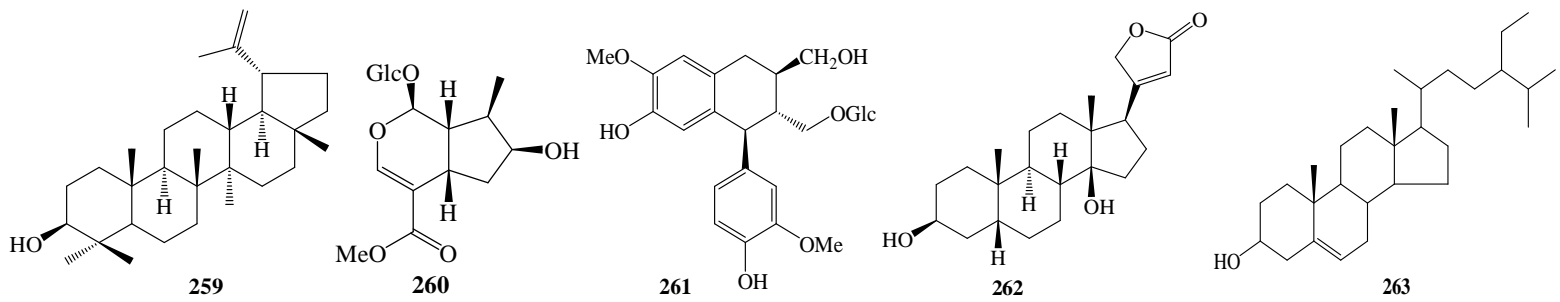

Figure 17: Structures of terpenoids and other compounds from Melodinus spps

dine (252). And alkaloid 251 was a quaternary ammonium and 252 possibly artifacts due to the use of $\mathrm{CH}_{2} \mathrm{Cl}_{2}$ as an extraction solvent [49].

\section{Diterpenoids, triterpenes and others compounds (Figure 17)}

A novel labdane diterpene, medigenin (253), Oacetyl-medigenin (254) and its glycoside, medinin (255), have been isolated from the ether extract of $M$. monogynus [74,75]. Four triterpenes, $\beta$-amyrin acetate (256), uvaol (257), monogynols A (258) and B (259), were isolated from $M$. henryi, $M$. monogynus and $M$. reticulatus [2,13,76-78]. One iridoid glycosides named loganin (260), a lignan glycoside, isolariciresinolmonoglucoside (261), two steroids, digitoxigenin (262) and $\beta$-sitosterol (263), were isolated from $M$. henryi, $M$. monogynus and $M$. morsei $[2,36,78]$.

\section{Extraction and isolation}

Four methods were used for extraction and isolation. Firstly, the air-dried sample was extracted with $\mathrm{EtOH}$ or $\mathrm{MeOH}$. The extract was partitioned between organic solvent and $\mathrm{HCl}$ solution. The acidic water-soluble materials, adjusted to $\mathrm{pH} 9$ - 10 with ammonia solution, were extracted with EtOAc, $\mathrm{MeOH}$ or $\mathrm{CHCl}_{3}$ to give an alkaloidal extract. Then, the extract was subjected to silica gel column chromatography (CC), TLC, MPLC with RP-18 gel CC, Sephadex LH-20 CC [27-29,32,34]. Secondly, M. fusiformis were extracted exhaustively with EtOH. After concentration, the material was obtained which was then dissolved in citric acid or tartaric acid, filtered and the solution adjusted to $\mathrm{pH} 5$ and 7 with $\mathrm{NH}_{4} \mathrm{OH}$, and then extracted with $\mathrm{CHCL}_{3}$, repeatedly. The extract was subjected to silica gel column chromatography (CC). Lastly, some dried Melodinus spps were ground and extracted with acetone or $\mathrm{EtOH}$. The extract was filtered and concentrated and the residue extracted with similarity principle. The extract was subjected to silica gel column chromatography (CC), $\mathrm{Et}_{2} \mathrm{O}$, $\mathrm{CHCL}_{3}$ and $\mathrm{MeOH}$ being used as eluents.

\section{Pharmacological aspects}

\section{Cytotoxic activity}

Continuous investigation of bioactive indole alkaloids from the genus Melodinus, include tests against seven human tumor cell lines, HL-60, SMMC-7721, A-549, MCF-7, SW480, SK-BR-3 and PANC-1.The results showed that these compounds exhibited stronger inhibitory activity with low $\mathrm{IC}_{50}$ than that of cisplatin (Table 6).

Pharmacological screening revealed 11hydroxytabersonine (24) displayed antitumor activity [17].

Compound 32, 11-hydroxy-14,15aepoxytabersonine, and rhazinilam (204) were the source of potent antitumor compounds against $P$ 338 cell and KB cell line $[15,79,80]$. Evaluated for cytotoxicity by using the WT cell, compound 207 exhibited moderate cytotoxic activity [35]. Demethylteuicausine (216), a new bisindole alkaloid was isolated from $M$. hemsleyanus, showed antitumor activities in pharmacological tests KB cell and HCT [6]. 
Table 6: Cytotoxicity of compounds $\left(\mathrm{IC}_{50}, \mu \mathrm{M}\right)$ from Melodinus spps

\begin{tabular}{ccccccccc}
\hline No. & HL-60 & $\begin{array}{c}\text { SMMC- } \\
\mathbf{7 7 2 1}\end{array}$ & A-549 & MCF-7 & SW480 & $\begin{array}{c}\text { SK- } \\
\text { BR-3 }\end{array}$ & PANC-1 & Ref \\
\hline $\mathbf{2 3}$ & 4.6 & 5.6 & 14.8 & 9.9 & 12.1 & - & - & {$[28]$} \\
$\mathbf{2 5}$ & 0.2 & 13.1 & 12.8 & 2.1 & 12.7 & - & - & {$[27]$} \\
$\mathbf{2 5}$ & 0.5 & 1.1 & 1.0 & 0.2 & 2.4 & - & - & {$[29]$} \\
$\mathbf{5 1}$ & 0.7 & 3.3 & 3.9 & 1.8 & 1.6 & - & - & {$[29]$} \\
$\mathbf{5 3}$ & 0.2 & 0.3 & 0.6 & 0.4 & 0.5 & - & - & {$[29]$} \\
$\mathbf{5 5}$ & 0.9 & 5.2 & 10.7 & - & - & 2.8 & 3.6 & {$[41]$} \\
$\mathbf{5 6}$ & 6.8 & 20.7 & 26.3 & 21.9 & 15.2 & - & - & {$[28]$} \\
$\mathbf{1 5 4}$ & 2.0 & 16.8 & 25.9 & - & - & 24.7 & & {$[48]$} \\
$\mathbf{1 7 1}$ & 15.48 & 19.08 & 40.0 & 14.24 & 13.29 & - & - & {$[53]$} \\
$\mathbf{2 1 1}$ & 0.66 & 2.73 & 3.01 & - & - & 3.63 & 3.77 & {$[71]$} \\
$\mathbf{2 1 2}$ & 2.77 & 18.13 & 11.07 & - & - & 23.22 & 23.41 & {$[71]$} \\
$\mathbf{2 1 7}$ & 3.0 & 8.5 & 9.1 & 10.0 & 14.8 & - & - & {$[27]$} \\
$\mathbf{2 1 8}$ & 1.1 & 3.2 & 4.8 & 2.9 & 1.4 & - & - & {$[27]$} \\
$\mathbf{2 2 2}$ & 2.84 & 11.54 & 20.07 & - & - & 15.41 & $>40$ & {$[71]$} \\
$\mathbf{2 3 0}$ & 2.53 & 7.40 & 14.70 & - & - & 7.78 & 14.45 & {$[71]$} \\
$\mathbf{2 3 1}$ & 0.1 & 3.0 & 5.0 & 2.7 & 5.7 & - & - & {$[27]$} \\
\hline
\end{tabular}

\section{Anti-inflammatory effects}

Two new compounds 187-188 exhibited significant, dose-dependent inhibition of the production of lipopolysaccharide (LPS)-induced NO, IL-6 and IL-8 in mice macrophages. The results suggested that these new quinoline alkaloids could be new potential candidates for development as anti-inflammatory agents [46].

\section{Other activities}

11,19R-dihydroxytabersonine (30) had significant anti-fertility activity. The results of spermicidal effect in vitro showed that spermicidal concentration of $0.2 \mathrm{mg} / \mathrm{ml}$ [15]. Rhazinilam (204) evaluated against the disassembly of microtubules into tubulin with $\mathrm{IC}_{50}$ value of 2 - 3 $\mu \mathrm{M}[79,80]$. Metabolism studies were conducted in order to investigate the reasons for the in vivo lack of activity of (-)-rhazinilam. The oxidative metabolism of (-)-rhazinilam were markedly less active than it in vitro, which might explain its in vivo inactivity [80].

\section{CONCLUSION}

Plants of the family Apocynaceae have been proven to be good sources of indole alkaloids, quinoline alkaloids, which originated from the condensation of tryptophan with secologanin. Some have been reported to display in vitro cytotoxicity against several human cancer cell lines, anti-inflammatory effects and antifertility activity. However, there still arise questions concerning the structure-activity relationships and elucidation of the action mechanism. There are 23 species of this genus whose isolation and activities have not previously been reported. Thus, much attention should be paid to the other
Melodinus species via further phytochemical, pharmacological and structure-activity relationship studies.

\section{ACKNOWLEDGEMENT}

This review was funded by National Natural Science Foundations of China (no. 31260085) and a grant (no. 2009CC018) from Yunnan Province of China for Basic Research in Social Development.

\section{REFERENCES}

1. Florae Reipublicae Popularis Sinicae, Tomus 63, Delectis Florae Reipublicae Popularis Sinicae Edita, Science Press, Beijing, 1977, pp.17.

2. Li CM, Wu SG, Tao GD, Zhong JY, You C, Zhou YL, Dong LY. Chemical constituents of Simao Shanchen (Melodinus henryi). Zhongcaoyao 1987; 18:52-53.

3. Zhong Hua Ben Cao, Shanghai Sentific \& Technical Publishers, Shanghai, 1999; 16: 293.

4. Boiteau P, Allorge L, Sevenet T. Adansonia 1976; 15:397-407.

5. Raymond H. Physiological properties of an Australian apocynaceous plant, Melodinus australis. Compt Rend 1956; 243:2175-2178.

6. Yan $K X$, Hong SL, Feng XZ. Demethyltenuicausine, a new bisindole alkaloid from Melodinus hemsleyanus. Acta Pharm Sin 1998; 33:597-599.

7. Au KS, Gray DE, Alkaloids of Melodinus suaveolens and their excretion as a common end-product in the rat. Biochem Pharmacol 1969; 18:1553-1557.

8. Zhang HM, Curran DP. A short total synthesis of ( \pm )epimeloscine and ( \pm )-meloscine enabled by a cascade radical annulation of a divinylcyclopropane. J Am Chem Soc 2011; 133:10376-10378 . 
9. Selig $P$, Bach $T$. Enantioselective total synthesis of the melodinus alkaloid (+)-meloscine. Angew Chem Int Ed 2008; 47:5082-5084.

10. Selig P, Herdtweck E, Bach T. Total synthesis of meloscine by a [2+2]-hotocycloaddition/ringexpansion route. Chem Eur J 2009; 15:3509-3525.

11. Sevenet T, Pusset J. The alkaloids: chemistry and pharmacology. Academic Press, New York, 1996; 48:34-38.

12. Szabo LF. Molecular interrelations in the melodinus alkaloids. Arkiboc 2007; vii:280-290.

13. Mehri H, Rochat $C$, Baassou S, Sevenet T, Plat M. Plant from new-caledonia. alkaloids of Melodinus reticulatus. Planta Med 1983; 48:72-76.

14. Zechis M, Lounkokobi J, Richard B, Plat M, Men-oliver LL, Sevenrt T, Pusset J. Alkaloids of Melodinus guillauminii. Phytochemistry 1984; 23:171-174.

15. He $X$, Zhou $Y$, Huang ZH. Study on the alkaloids of Melodinus fusiformis. Acta Chim Sinica 1992; 50:96101.

16. He YL, Chen WM, Feng XZ. The alkaloids of Melodinus morsei. Phytochemstry 1994;37: 1055-1057.

17. Yan KX, Feng XZ. Studies on the chemical constituents of hemsley Melodinus (Melodinu shemsleyanus). Tradit Herb Drugs 1998; 29:793-795.

18. Linde HHA. Die alkaloide aus Melodinus australis ( $F$. Mueller) Pierre (Apocynaceae). Helv Chim Acta 1965; 48, 1822-1842.

19. Mehri H, Plat M, Potier P. Apocynaceae of new caledonia, V. Melodinus scandens. Isolation of 10 momomeric alkaloids. description of two new alkaloids, $N$-oxyepimeloscine and meloscandonine. Ann Pharm Fr 1971; 29:291-296.

20. Mehri $H$, Koch $M$, Plat $M$, Potier P. Plants of new caledonia, XII. alkaloids of the leaves of Melodinus balansae (Apocynaceae). Ann Pharm Fr 1972; 30:643-650.

21. Mehri H, Rabaron A, Sevenet T, Plat M. Alcaloides de Melodinus balansae var. paucivenosus. Phytochemistry 1978; 17:1451-1452.

22. Le ML, Levy J, Sevenet T, Plat M. Plants of new caledonia, alkaloids from Melodinus polyadenus Baill. Ann Pharm Fr 1981; 39:369-373.

23. Mehri $H$, Sciamama $S$, Plat $M$, Sevenet $T$, Pusset J. Plants from new caledonia, alkaloids from Melodinus phylliraeoides Labill. Ann Pharm Fr 1984; 42:145148.

24. Batchily F, Baassou S, Mehri H, Plat M, Sevenet T, Pusset J. Plants from new caledonia, part 90. alkaloids from Melodinus insulae-pinorum Boiteau. Ann Pharm Fr 1985; 43:359-364.

25. Guo LW, Zhou YL. Alkaloid from Melodinus hemsleyanus. Phytochemistry 1993; 34:563-566.

26. Lien LN, Dien PG, Pais M. Alkaloid from Melodinus oblongus(Apocynaceae). Tap Chi Hoa Hoc 2002; 40:47-51.
27. Feng $T$, Li $Y$, Wang $Y Y$, Cai XH, LiU YP, Luo XD. Cytotoxic indole alkaloids from Melodinus tenuicaudatus. J Nat Prod 2010; 73:1075-1079.

28. Cai $X H$, Jiang $H$, Li Y, Cheng GG, Liu YP, Feng T, Luo $X D$. Cytotoxic indole alkaloids from Melodinus fusiformis and M. morsei. Chin J Nat Med 2011; 9:259-263.

29. Liu YP, Li Y, Cai XH, Li XY, Kong LM, Cheng GG, Luo $X D$. Melodinines $M-U$, cytotoxic alkaloids from Melodinus suaveolens. J Nat Prod 2012; 75:220-224.

30. Lien LN, Dien PG, Pais M. Alkaloid from Melodinus oblongus (Apocynaceae). Tap Chi Hoa Hoc 2002; 40:79-85.

31. Baassou S, Mehri H, Rabaron A, Plat M, Sevenet $T$. Plants of new caledonia, alkaloids of Melodinus celastroides Baill. Ann Pharm Fr 1981; 39:167-170.

32. Cai XH, Li Y, Liu YP, Li XN, Bao MF, Luo XD. Alkaloids from Melodinus yunnanensis. Phytochemistry 2012; 83:116-124.

33. Linde HH. Alkaloid from Melodinus australis. 2. structure of refractidine and pyrifoline. Pharm Acta Helv 1970; 45:248-253.

34. Kitajima $M$, Ohara $S$, Kogure $N$, Wu $Y Q$, Zhang $R P$, Takayama $H$. New indole alkaloids from Melodinus henryi. Heterocycles 2012; 85:1949-1959.

35. Zhou H, He HP, Wang YH, Hao XJ. A novel alkaloid from Melodinus henryi. Helv Chim Acta 2010; 93:20302032.

36. He YL, Chen WM, Feng XZ. Chemical constituent from Melodinus morsei. Chin Tradit Herb Drugs 1993; 24:623-265.

37. Ye JH, Zhou YL, Huang ZH, Picot F. Alkaloids from Melodinus suaveolens. Phytochemistry 1991; 30:3168-3170.

38. Baassou S, Mehri H, Plat M. Alcaloides de Melodinus aeneus. Phytochemistry 1978; 17:1449-1450.

39. Rabaron A, Plat M, Potier P. Isolement dela dehydro-14 isoeburnamine de Melodinus celastroides. Phytochemistry 1973; 12:2537-2539.

40. Rabaron A, Mehri H, Sevenet T, Plat M. Alcaloides de Melodinus celastroides. Phytochemistry 1978; 17:1452-1453.

41. Feng $T, L i Y$, LiU YP, Cai $X H$, Wang $Y Y$, Luo $X D$. Melotenine $A$, a cytotoxic monoterpenoid indole alkaloid from Melodinus tenuicaudatus. Org Lett 2010; 12:968-971.

42. Li CM, Tao GD, Wu SG, Zhou YL. Indole alkaloid of Melodinus henryi Craib. Acta Botan Sin 1989; 31:792-797.

43. Zhou YL, Ye JH, Li ZM, Huang ZH. Study on the alkaloids of Melodinus tenuicaudatus. Planta Med 1988; 54:315-317.

44. He YL, Chen WM, Feng XZ. Melomorsine, a new dimeric indoline from Melodinus morsei. J Nat Prod 1994; 57:411-414.

45. Yan KX. Chemical constituent from Melodinus axillaris and M. Hemsleyanus [dissertation]. [China]: Peking Union Medical College, 1997 May. 1- 58.

Trop J Pharm Res, December 2015; 14(12): 2342 
46. Lu F, Liu P, Yin SS, Zhang J, Wang J. Two new alkaloids from Melodinus suaveolens. Nat Prod Commun 2014; 9:1445-1447.

47. He YL, Chen WM, Feng XZ. Two new indole alkaloid of Melodinus morsei. Chin Chem Lett 1992; 3:715-718.

48. Feng $T$, Cai XH, LiU YP, Li $Y$, Wang $Y Y$, Luo XD. Melodinines $A-G$, monoterpenoid indole alkaloids from Melodinus henryi. J Nat Prod 2010; 73:22-26.

49. Mehri H, Baasou S, Flat M. Methylene-10,10'-bis[(+)Nanorvallesamidine], methylene- $\mathrm{Nb}, \mathrm{Nb}^{\prime}[\mathrm{bis}$ (+)meloninium] dichloride, and the chlorides of Nb'chloromethyl celastromelinium and celastromelidinium: dimeric alkaloids and possible extraction artifacts from Melodinus celastroides. J Nat Prod 1991; 54:372-379.

50. Rabaron A, Plat M. Plants of new caledonia, XXVIII. alkaloids from Melodinus celastroides. Planta Med 1973; 7:319-324.

51. Li CM, Yang HC, Wu SG, Sun HD. Indole alkaloids from stem bark of Melodinus khasianus. Acta Botan Yunnan 1996; 18:356-358.

52. Zhang YW, Yang R, Cheng Q, Ofuji K. Henrycinols $A$ and $B$, two novel indole alkaloids isolated from Melodinus henryi. Helv Chim Acta 2003, 86:415-419.

53. Cai XH, Li Y, Su J, Liu YP, Li XN, Luo XD. Novel indole and quinoline alkaloids from Melodinus yunnanensis. Nat Prod Bioprospect 2011; 1:25-28.

54. Li CM, Tao GD, Zhou YL. A new indole alkaloid from leaves of Melodinus henryi. Acta Botan Yunnan 1992; 14:32,66.

55. Zhang TT, Liu ZW, Wang WJ, Tong TB, Xu FF, Yuan JQ, Liu B, Zhang XQ, Ye WC. Alkaloids from Melodinus suaveolens. Heterocycles 2013, 87:2047-2052.

56. Hu WL, Hesse M. The alkaloids of Melodinus acutiflorus. rhyzicine N-Oxide. Planta Med 1988; 54:235-236.

57. Feng T, Cai XH, Li Y, Wang YY, Liu YP, Xie MJ, Luo XD. Melohenines $A$ and $B$, two unprecedented alkaloids from Melodinus henryi. Org Lett 2009; 11:4834-4837.

58. Bernauer K, Englert G, Vetter W, Weiss E. Constitution of the melodinus alkaloids (+)-meloscine, (+)epimeloscine, and (+)-scandine. Helv Chim Acta 1969; 52:1886-1905.

59. Li CM, Zhang HL, Wu SG, Sun HD. Quinolinic melodinus alkaloids from stem bark Melodinus khasianus. Acta Botan Yunnan 1994; 16:315-317.

60. Daudon M, Mehri H, Plat M, Hagaman EW, Schell FM, Wenkert E. Carbon-13 nuclear magnetic resonance spectroscopy of naturally occurring substances. XXXIV. monomeric quinolinic melodinus alkaloids. $J$ Org Chem 1975; 40: 2838-2339.

61. Cannon JR, Croft KD, Matsuki Y, Patrick VA, Toia RT, White AH. Crystal structure and absolute configuration of $(+)$-scandine hydrobromide. Aust $J$ Chem 1982; 35: 1655-1664.

62. Gu JM, Hu XR. Sandine: a catharanthus-derived alkaloid. Acta Cryst 2006; E62:4775-4777.

63. Ma K, Wang JS, Luo J, Kong LY. Six new alkaloids from Melodinus henryi. Fitoterapia, 2015, 100:133-138.
64. Mehri $H$, Diallo AO, Plat M. An alkaloid from leaves of Melodinus scandens. Phytochemistry 1995; 40:10051006.

65. Rodier $N$, Mauguen $Y$, Hachem-Mehri M, Plat $M$. Structure cristalline de la me1oscandonine, C20H20N2O2: alcaloide du Melodinus seandens Forst. Acta Cryst 1978; B34:232-237.

66. Plat M, Hachem-Mehri M, Koch M, Scheidegger U, Potier $P$. Structure et stereochimie de la me1oscandonine, alcaloide du Melodinus seandens Forst. Tetrahedron Lett 1970; 39:3395-3398.

67. Hu WL, Zhu JP, Umberto $P$, Roland $P$, Manfred $H$. Revision of the structures of rhazicine and rhazimine, two alkaloids from Melodinus acutiflorus. Phytochemistry 1987; 26:2625-2630.

68. Abraham DJ, Rosenstein RD, Lyon RL, Fong HHS. The structure elucidation of rhazinilam, a new class of alkaloids from the apocynaceae, by $X$-ray analysis. Tetrahedron Lett 1972; 13:909-912.

69. Liu YP, Zhao YL, Feng T, Cheng GG, Zhang BH, Li Y, Cai $X H$, Luo $X D$. Melosuavines $A-H$, cytotoxic bisindole alkaloid derivatives from Melodinus suaveolens. J Nat Prod, 2013; 76:2322-2329.

70. Yan KX, Feng XZ. Melaxillarinine, a new bisindole alkaloid from Melodinus axillaris. Chin Chem Lett 1997; 8:313-314.

71. Luo $X D$, Feng $T$, Li $Y$, Wang $Y Y$, Cai XH. Extraction method of melonine di-indole compounds from Chinese medicine Melodinus and their medical application as antitumor agents. Faming Zhuanli Shenqing. CN. Pat. 101, 704, 828 (Chem Abstr 2010, 153: 45657)

72. Mehri $H$, Flat $M$. The structure of scandomelidine, bisindole alkaloid from Melodinus scandens. J Nat Prod 1992; 55:241-244.

73. Daudon M, Mehri H, Plat M, Hagaman W, Wenkert E. Carbon-13 nuclear magnetic resonance spectroscopy of naturally occurring substances. 48. dimeric quinolinic melodinus alkaloids. J Org Chem 1976; 41:3275-3278.

74. Bhntnngar SOD, Khare MP. Herzwirksame glykoside aus Melodinus monogynus Roxb.Chem Ber 1968, 101:2084-2095.

75. Sethi A, Khare A, Khare MP. A labdane diterpene and its glycoside from Melodinus monogynus. Phytochrmistry 1988; 27:2255-2259.

76. Chatterji SK, Sharma VN, Dhar ML. Chemical examination of the root bark of Melodinus monogynus. I. J Sci Ind Research Inst(India), 1954; 13:546-549.

77. Chatterji SK, Anand N, Dhar ML. Chemical examination of Melodinus monogynus. II Identifacation of monogunol $A$ and monogunol B. J Sci Ind Research Inst(India), 1959; 18B:262-263.

78. Mitscher LA, Vorperian EN. Triterpenes from Melodinus australzs. Phytochrmistry 1971; 10:1687-1688.

79. Dupont C, Gueanard D, Tchertanov L, Thoret S, Gueritte F. D-ring substituted rhazinilam analogues:

Trop J Pharm Res, December 2015; 14(12): 2343 
semisynthesis and evaluation of antitubulin activity. Bioorg Med Chem 1999; 7:29612969.

80. Decor A, Bellocq D, Thoison O, Lekieffre N, Chiaroni A, Ouazzani J, Cresteil T, Gueritte $F$ and Baudoin O. In vitro oxidative metabolism study of (-)-rhazinilam. Bioorg Med Chem 2006; 14:1558-1564. 